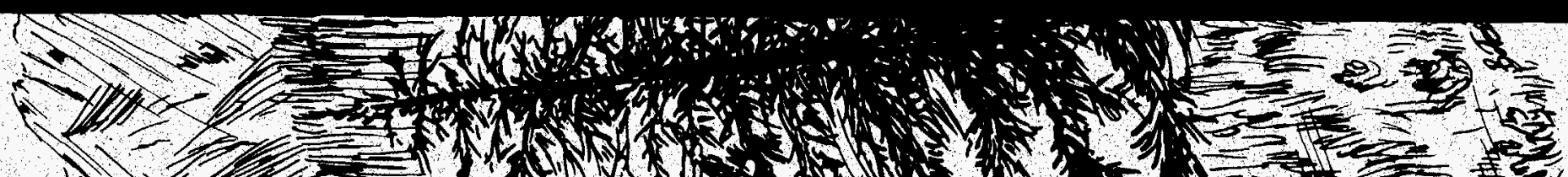

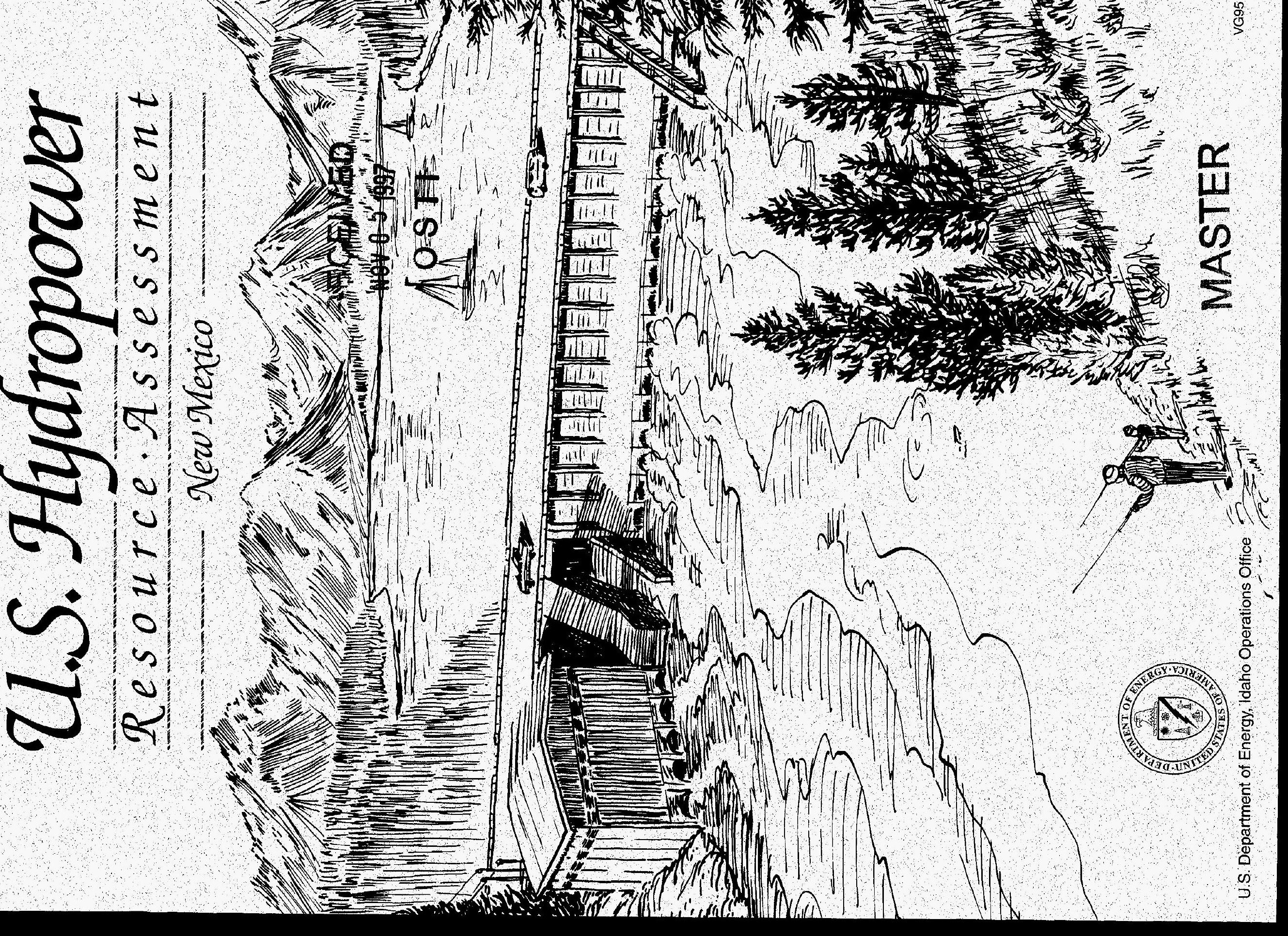




\title{
U.S. Hydropower Resource Assessment for New Mexico
}

\author{
Prepared by: \\ Alison M. Conner \\ James E. Francfort \\ Project Manager: \\ Ben N. Rinehart
}

Published March 1997

\begin{abstract}
Idaho National Engineering Laboratory Renewable Energy Products Department Lockheed Martin Idaho Technologies Company Idaho Falls, Idaho 83415
\end{abstract}




\section{DISCLAMERT}

Portions of this docoment moy be illegibie in electronic image products. Images are produced from the best arailable original document 


\begin{abstract}
The U.S. Department of Energy is developing an estimate of the undeveloped hydropower potential in the United States. The Hydropower Evaluation Software (HES) is a computer model that was developed by the Idaho National Engineering Laboratory for this purpose. HES measures the undeveloped hydropower resources available in the United States, using uniform criteria for measurement. The software was developed and tested using hydropower information and data provided by the Southwestern Power Administration. It is a menu-driven program that allows the personal computer user to assign environmental attributes to potential hydropower sites, calculate development suitability factors for each site based on the environmental attributes present, and generate reports based on these suitability factors. This report describes the resource assessment results for the State of New Mexico.
\end{abstract}




\section{ACKNOWLEDGMENTS}

The authors thank Peggy A. M. Brookshier, John V. Flynn, and Wayne Belgrave of the U.S. Department of Energy and Michael McDiarmid of the State of New Mexico for their active participation and timely comments. 


\section{CONTENTS}

ABSTRACT

ACKNOWLEDGMENTS

INTRODUCTION

Model Development

Model Goal.

Dam Status

ASSESSMENT RESULTS

Summary Results

Detailed Results

OBTAINING INDIVIDUAL STATE INFORMATION

ADDITIONAL HYDROPOWER EVALUATION SOFTWARE INFORMATION

REFERENCES

Appendix A-Summary Report

A-1

Appendix B-River Basins Report

B-1

Appendix C-New Mexico Sites List

C-1

Appendix D-Individual Resource Database List

D-1

\section{FIGURES}

1. Number of sites, by capacity groups, with HES-modeled undeveloped hydropower potential ....

2. The nonmodeled and HES-modeled undeveloped hydropower potential

3. The number of sites with undeveloped hydropower potential and the total megawatts of HES-modeled undeveloped hydropower potential.

4. The Conchas Dam is an example of a developed site in New Mexico without current hydropower generation.

5. The Ute Dam is another example of a developed site in New Mexico without current hydropower generation.

6. Number of sites with undeveloped hydropower potential in the New Mexico river basins

7. Megawatts of HES-modeled undeveloped hydropower potential in the New Mexico river basins 


\section{TABLES}

1. Undeveloped hydropower potential summaries for New Mexico... 


\section{U.S. Hydropower Resource Assessment for New Mexico}

\section{INTRODUCTION}

In June 1989, the U.S. Department of Energy initiated the development of a National Energy Strategy to identify the energy resources available to support the expanding demand for energy in the United States. Public hearings conducted as part of the strategy development process indicated that undeveloped hydropower resources were not well defined. As a result, the Department of Energy established an interagency Hydropower Resource Assessment Team to ascertain the undeveloped hydropower potential. In connection with these efforts by the Department of Energy, the Idaho National Engineering Laboratory designed the Hydropower Evaluation Software (HES), which has been used to perform a resource assessment of the undeveloped conventional hydropower potential in over 30 states. This report presents the results of the hydropower resource assessment for the State of New Mexico. Undeveloped pumped storage hydropower potential is not included.

The HES was developed as a tool to measure undeveloped hydropower potential regionally or by state. The software is not intended to provide precise development factors for individual sites, but to provide regional or state totals. Because the software was developed as a generic measurement tool encompassing national issues, regional and state totals must be considered judiciously; various local issues may skew undeveloped hydropower potential totals. The information for the resource assessment was compiled from the Federal Energy Regulatory Commission's Hydroelectric Power Resources Assessment database and several other sources. Refer to DOE/ID-10338, the User's Manual (Francfort, Matthews, Rinehart 1991) for the specifics of the software and to DOE/ID10430.1, the Status Report (Conner, Francfort, Rinehart 1996) for an overview of all resource assessment activities to date.

\section{Model Development}

Hydropower Evaluation Software, both a probability-factor computer model and a database, is a menu-driven program that is intended to be user-friendly. Computer screens and report-generation capabilities were developed to meet the needs of users nationwide. The software uses environmental attribute data to generate an overall project environmental suitability factor (PESF) between 0.1 and 0.9 , where 0.9 indicates the highest likelihood of development and 0.1 indicates the lowest likelihood of development. The suitability factors are dependent on the unique environmental attributes of each potential site. They reflect the considerations that (a) environmental concerns can make a potential site unacceptable, prohibiting its development (for a suitability factor of 0.1 ), or (b) if there are no environmental concerns, there is no negative effect on the likelihood of site development (for a suitability factor of 0.9 ). A combination of attributes can result in a lower suitability factor because multiple environmental considerations would reduce the likelihood that a site may be developed to its physical potential.

\section{Model Goal}

The goal of the HES is to assemble an accurate resource database of all sites with undeveloped hydropower potential in the United States for use as a planning tool to determine the viable national hydropower potential. Undeveloped hydropower potential is not limited to the development of new sites; it also includes the development of additional hydropower-generating capacity at sites that currently have hydropower, but are not developed to their full potential. This undeveloped hydropower potential is a source of nonpolluting, renewable energy available to meet the growing power needs of the United States. The HES should help make this goal obtainable and ensure a set of uniform criteria for national assessment. 


\section{Dam Status}

The effects of environmental attributes vary by dam status. The dam status classifications used are as follows

$\mathrm{W}=$ Developed hydropower site with current power generation, but the total hydropower potential has not been fully developed. Only the undeveloped hydropower potential is discussed in this report.

W/O $=$ Developed site without current power generation. The site has some type of developed impoundment or diversion structure, but no developed hydropower generating capability.

$\mathrm{U}=$ Undeveloped site. The site does not have power generation capability nor a developed impoundment or diversion structure.

\section{ASSESSMENT RESULTS}

\section{Summary Results}

A total of 22 sites (Table 1) have been identified and assessed for their undeveloped hydropower potential. The HES results for individual site capacities range from 9 kilowatts $(\mathrm{kW})$ to 11 megawatts (MW). The majority of the sites $(64 \%)$ have potential capacities of less than $1 \mathrm{MW}$ (Figure 1).

The nonmodeled undeveloped

hydropower potential total for New Mexico was identified as $90 \mathrm{MW}$. The HES results lowers this estimate about $61 \%$ to $35 \mathrm{MW}$. The greatest reduction in undeveloped hydropower potential, by MW, occurs at sites with no power generation capability, nor any type of structure (undeveloped site category). These sites have an HES-modeled undeveloped hydropower potential of $5 \mathrm{MW}$, a $26 \mathrm{MW}$ reduction in the estimated undeveloped hydropower potential (Figure 2). The developed sites with power generation capability have the least percentage decrease in modeled undeveloped potential capacity. The unadjusted potential for the sites with power is $11 \mathrm{MW}$, and the modeled capacity is $6 \mathrm{MW}$, a $45 \%$ decrease in capacity (Figure 2 ). Figure 3 illustrates that the undeveloped sites have the smallest HES-modeled average capacity per site $(0.6 \mathrm{MW})$, and the developed sites with power have the highest HES-modeled average capacity per site ( $3 \mathrm{MW}$ ). Figures 4 and 5 show examples of two sites with undeveloped hydropower potential in New Mexico.

The 22 identified sites are located within 7 major river basins. The number of sites per major river basin ranges from 1 in the Rio Penasco River Basin, to 7 in the Upper Rio Grande River Basin (Figure 6). The Upper Rio Grande River Basin has the most undeveloped hydropower potential (23 MW) of the New Mexico river basins (Figure 7). It should be noted that about one-half of $23 \mathrm{MW}$ of undeveloped hydropower potential is located at one site.

Table 1.Undeveloped hydropower potential summaries for New Mexico. The table contains the nonmodeled undeveloped nameplate potential and the HES-modeled undeveloped hydropower potential totals.

Nameplate potential HES-modeled potential

Number of projects $\quad(\mathrm{MW}) \quad$ (MW)

\begin{tabular}{lrrr} 
With Power & 2 & 11.0 & 5.5 \\
W/O Power & 12 & 47.9 & 24.3 \\
Undeveloped & 8 & 31.3 & 5.0 \\
\hline State Total & 22 & 90.2 & 34.8 \\
\hline
\end{tabular}




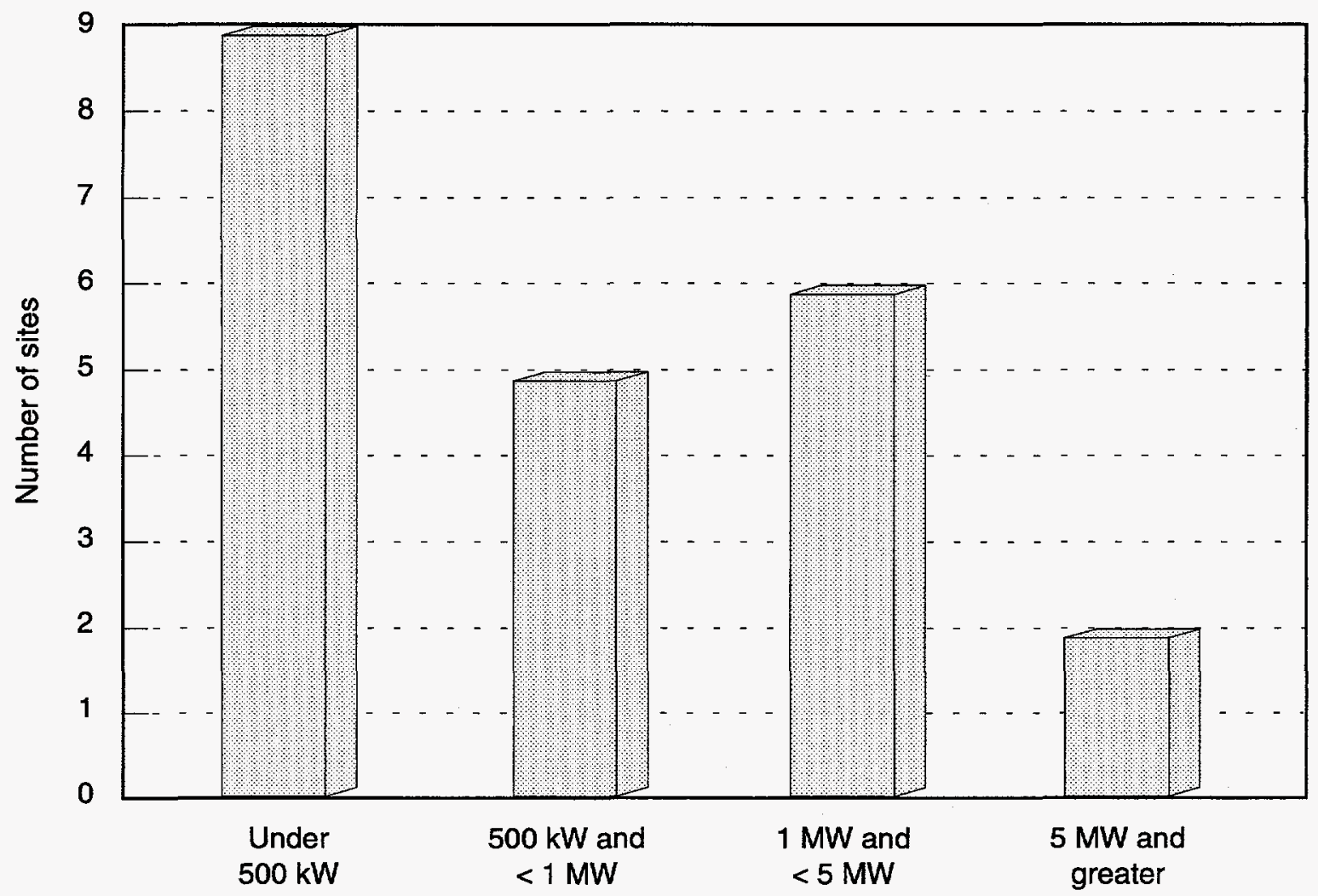

Figure 1. Number of sites, by capacity groups, with HES-modeled undeveloped hydropower potential.

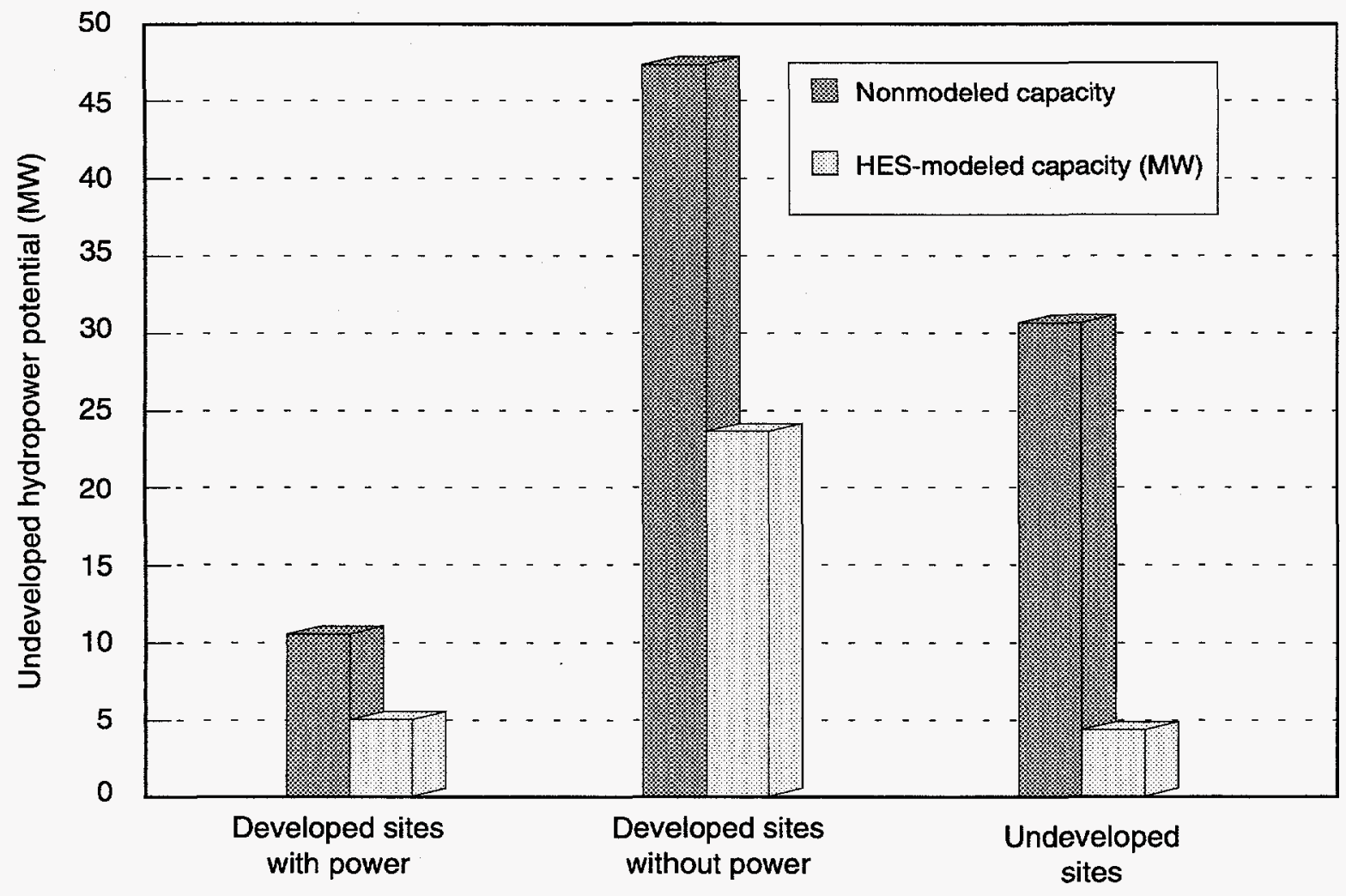

Figure 2. The nonmodeled and HES-modeled undeveloped hydropower potential. 


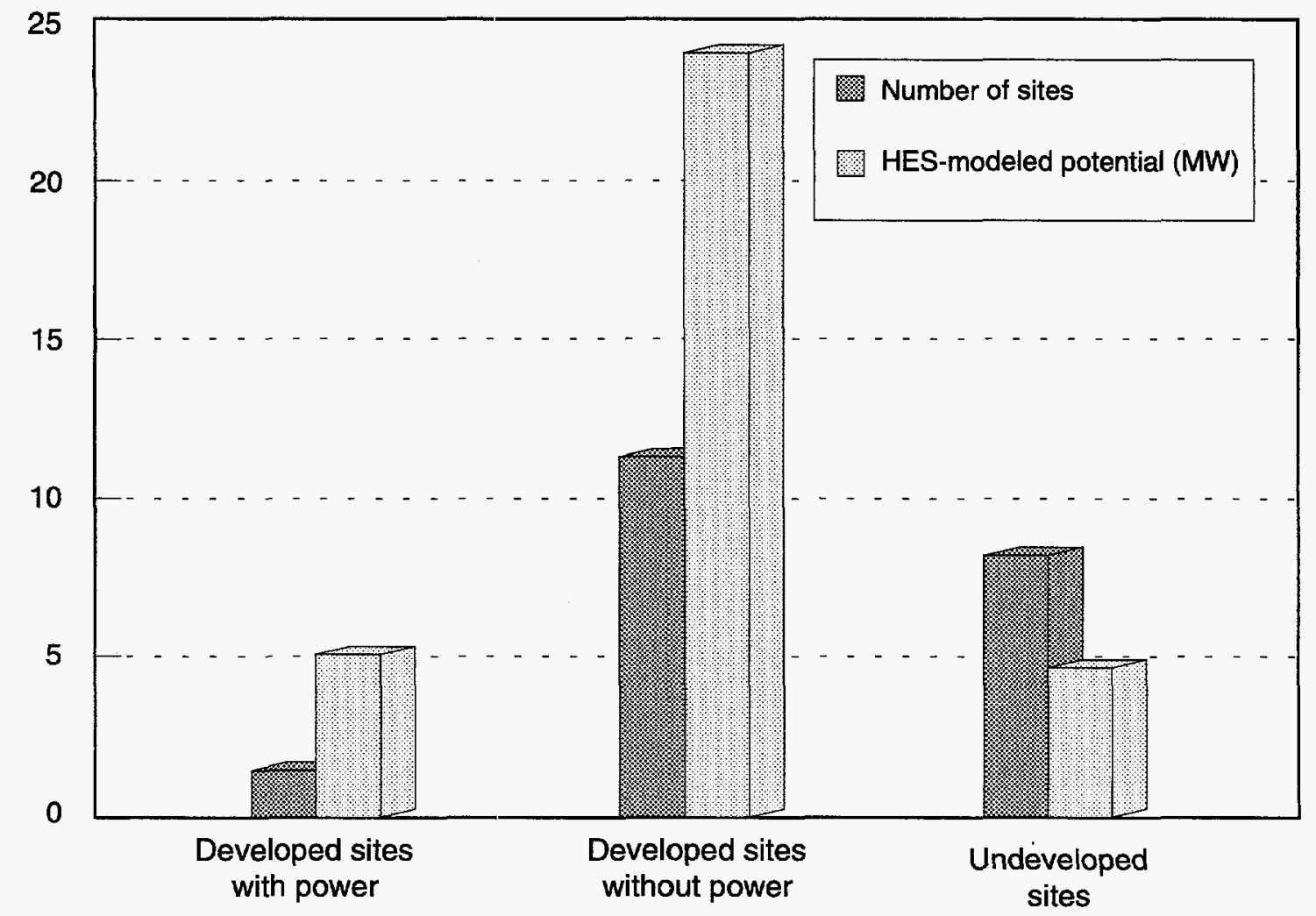

Figure 3. The number of sites with undeveloped hydropower potential and the total megawatts of HESmodeled undeveloped hydropower potential.

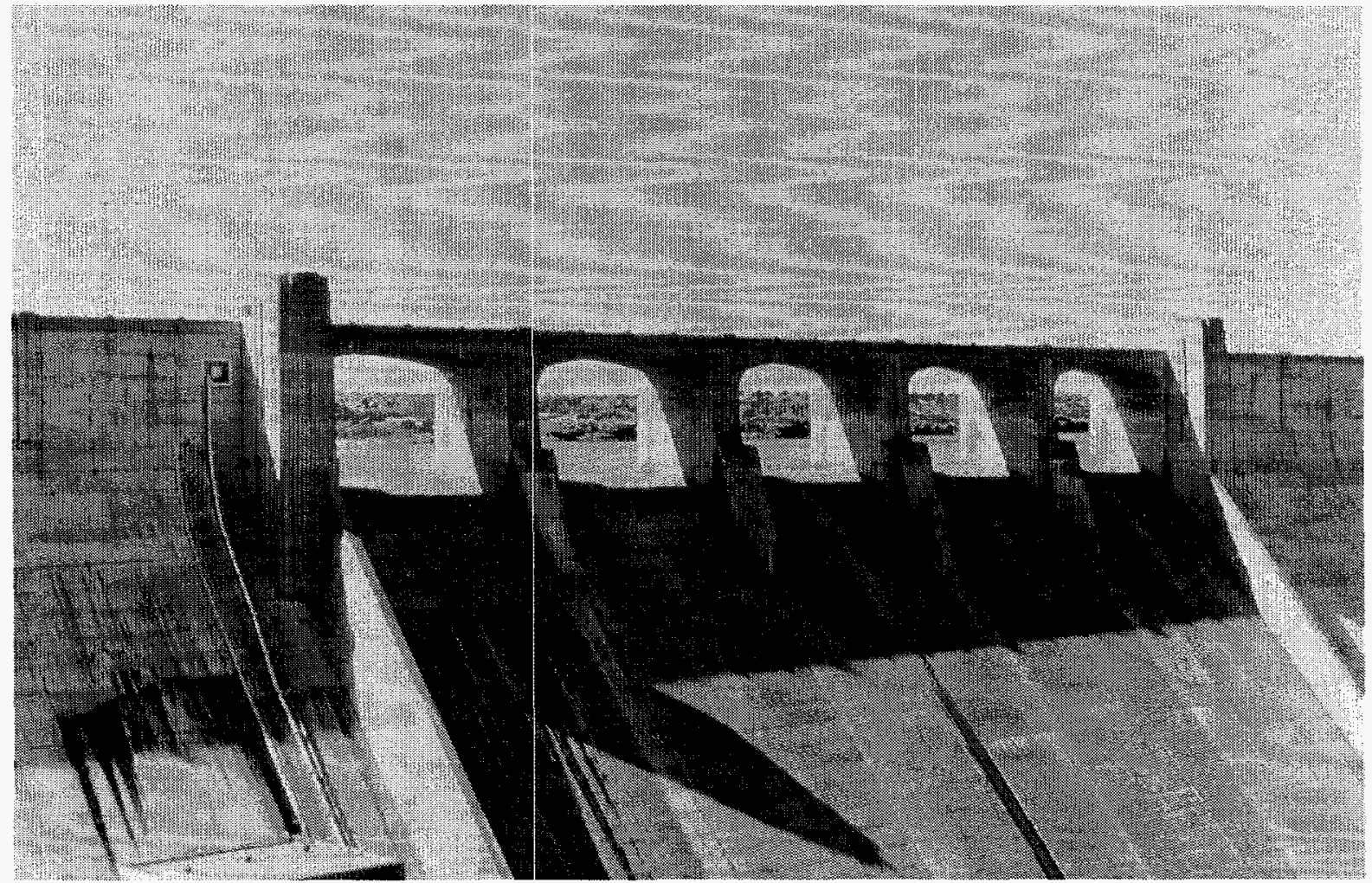

Figure 4. The Conchas Dam is an example of a developed site in New Mexico without current hydropower generation. It is located on the Canadian River near Tucumcari, New Mexico, and has the undeveloped hydropower potential of $2,078 \mathrm{~kW}$. 


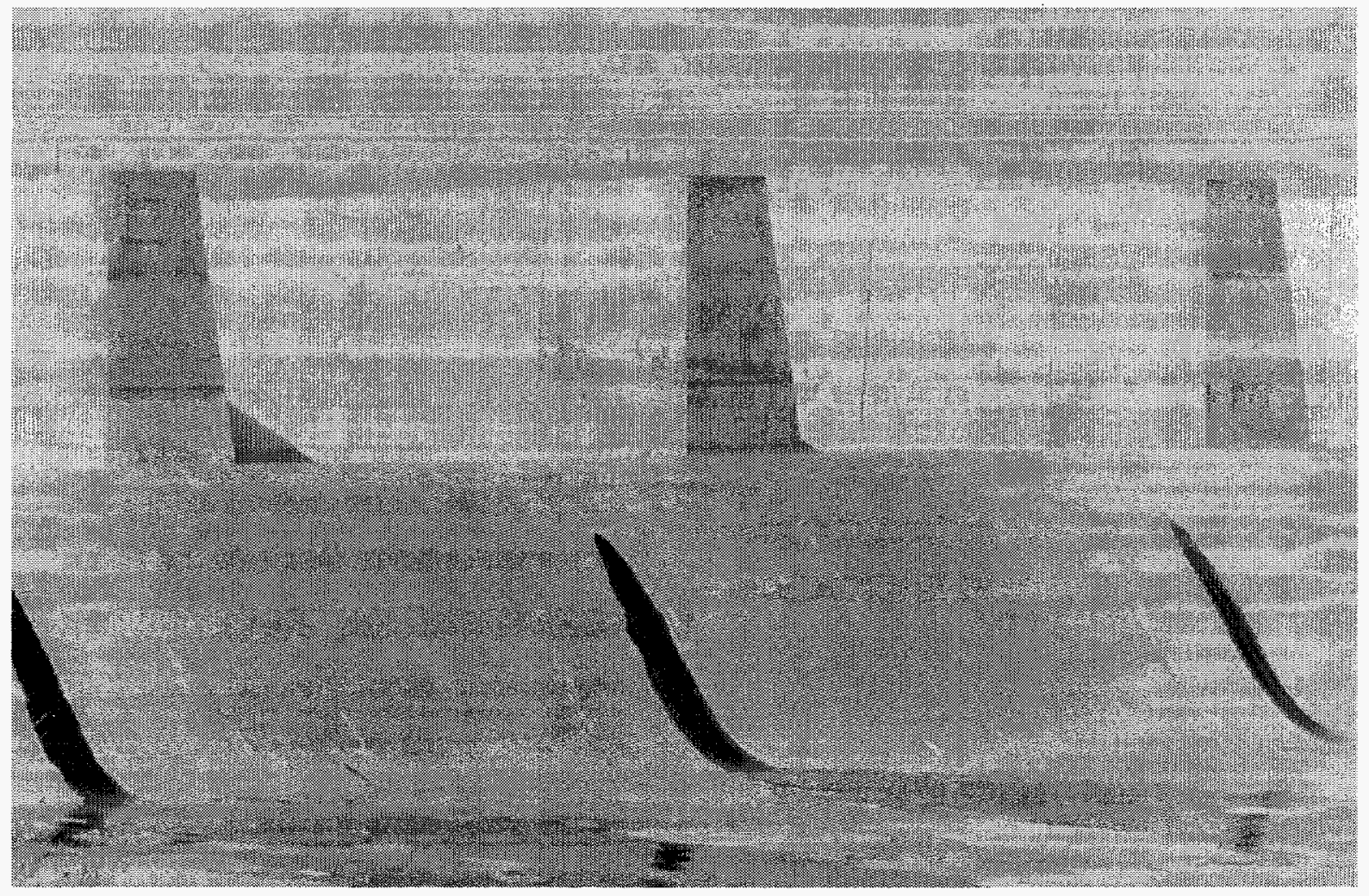

Figure 5. The Ute Dam is another example of a developed site in New Mexico without current hydropower generation. It is located on the Canadian River near Tucumcari, New Mexico, and has the undeveloped hydropower potential of $2,640 \mathrm{~kW}$.

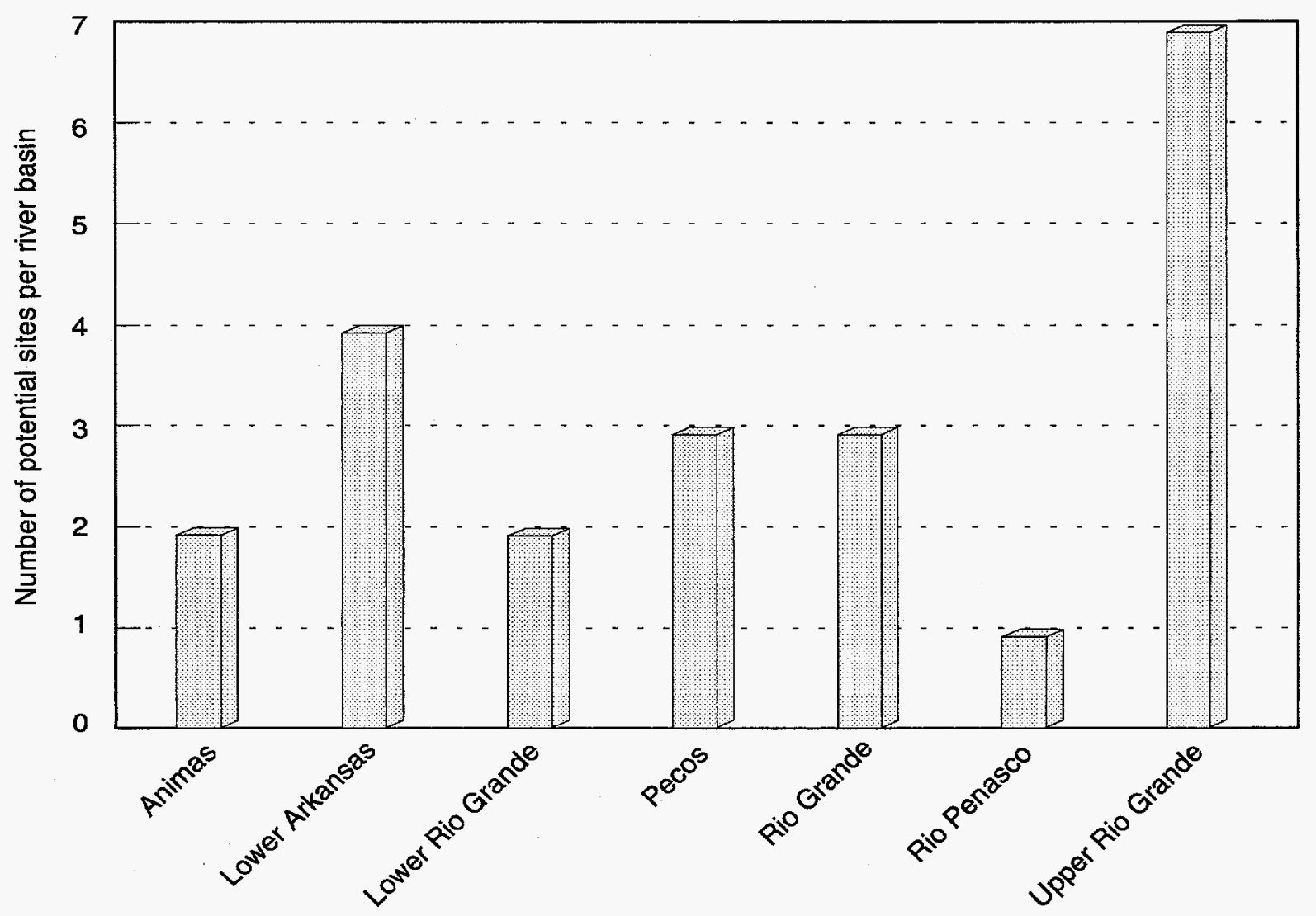

Figure 6. Number of sites with undeveloped hydropower potential in the New Mexico river basins. 


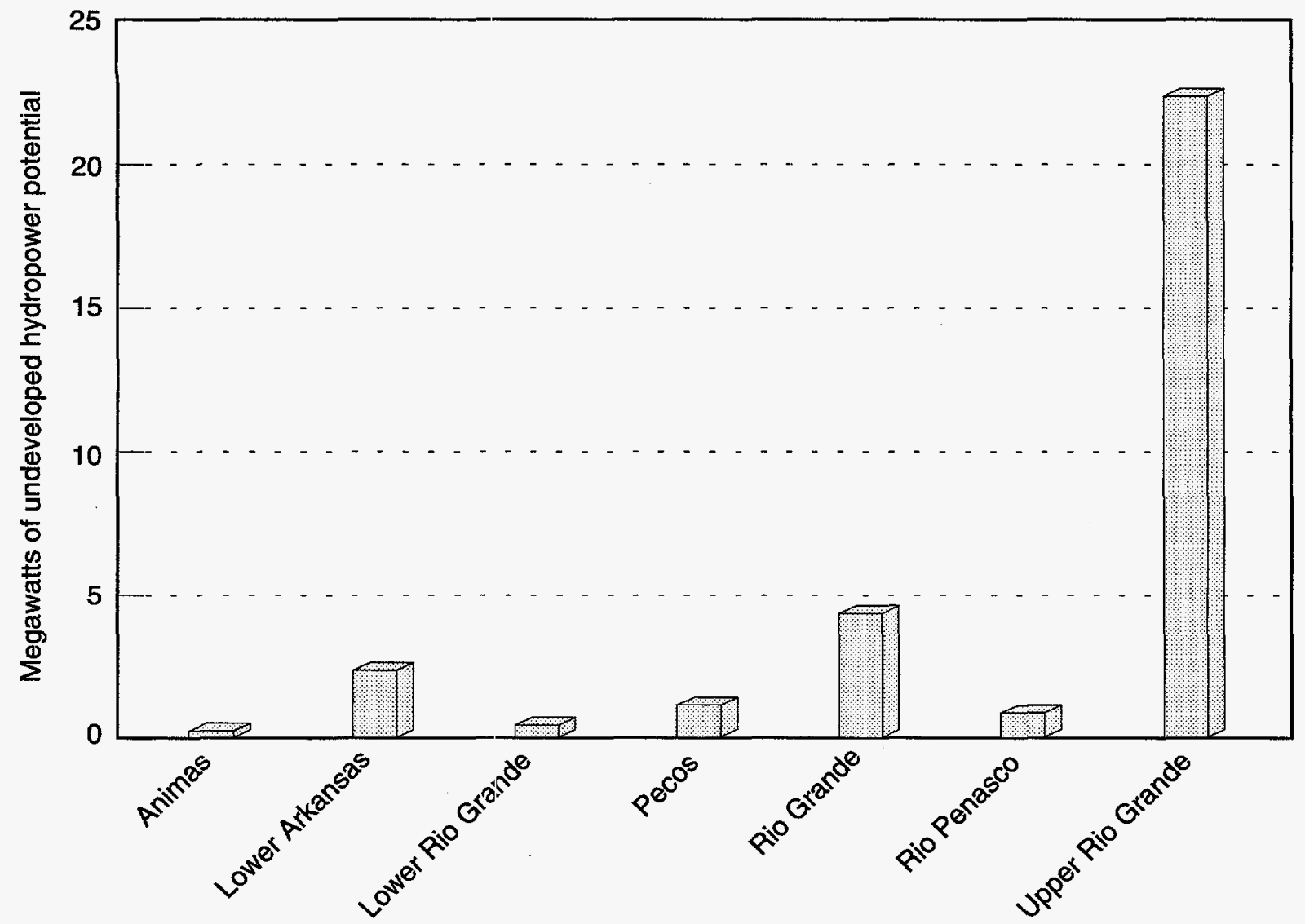

Figure 7. Megawatts of HES-modeled undeveloped hydropower potential in the New Mexico river basins.

\section{DETAILED RESULTS}

The appendices contain, in the form of HES-generated reports, detailed information about the undeveloped hydropower potential in New Mexico. The appendices contain the following information:

Appendix A summarizes the undeveloped hydropower potential by dam status groups. The number of sites, nonmodeled undeveloped hydropower potential, and HES-modeled undeveloped hydropower potential is provided based on the dam status.

Appendix $B$ provides the hydropower resource assessment by river basin, which includes the project number, project name, stream name, dam status, nonmodeled undeveloped hydropower potential, and the HES-modeled undeveloped hydropower potential for each of the individual sites. Subtotals are provided for each river basin.
Appendix $\mathrm{C}$ provides the hydropower resource assessment by FERC number, which includes the project numbers, plant name, stream name, if a site is Federally owned, nonmodeled undeveloped hydropower potential, and HESmodeled undeveloped hydropower potential. The sites are grouped by dam status.

Appendix D contains a resource database list for each of the 22 sites in New Mexico. Information includes plant name, stream, state, county, river basin and owner names, project number, nameplate and HES-modeled undeveloped hydropower potential, the unit and plant types, dam status, latitude, longitude, and the environmental factors that the HES uses to determine the project environmental suitability factor.

\section{OBTAINING INDIVIDUAL STATE INFORMATION}

Additional copies of the hydropower resource assessment results for individual states are available and can be obtained by writing or 
calling the authors or the National Technical Information Service (NTIS).

Telephone Orders-(703) 487-4650. NTIS sales desk and customer services are available between 8:30 a.m. and 5:00 p.m., EST.

Fax-(703) 321-8547. Customers may fax their orders to NTIS. These orders may be charged to a NTIS deposit account, American Express, VISA, or MasterCard.

Mail Orders-Mail orders should be sent to National Technical Information Service, Document Sales, 5285 Port Royal Road, Springfield, VA 22161. Call the sales desk for prices before placing an order.

Method of Payment-Customers may pay for reports (and other NTIS products and services) by (a) credit card (American Express, Visa or MasterCard); (b) check or money order on a United States bank payable to NTIS; (c) an NTIS deposit account; or, (d) by asking to be billed (add $\$ 7.50$ per order), United States, Canada, and Mexico, only.

Handling Fee-A $\$ 3.00$ handling fee per total order applies to orders from the United States, Canada, and Mexico. Handling charges do not apply to rush order service or pick-up orders.

Postage and Shipping-Orders are shipped first class mail, or equivalent, to addresses in the United States, Canada, and Mexico.

Order Turnaround Time-Orders for technical reports generally are shipped within 2 to 8 days of receipt. For faster service, NTIS offers rush order service.

Rush Order Service-Call 1-800-533-NTIS. In Virginia, Canada, and Mexico call (703) 487 4700. For NTIS rush order service add $\$ 15$ per item. This guarantees that an order will be processed through NTIS within 24 hours of its receipt. These orders receive immediate, individ- ual attention. The items ordered are delivered by first call mail. Call NTIS for information on rush order service for computer products.

For Help in Tracing an Order-Call (703) 487-4650 and request the customer service option.

\section{ADDITIONAL HYDROPOWER EVALUATION SOFTWARE INFORMATION}

Additional information concerning the HES can be obtained by contacting Ben Rinehart or Jim Francfort at the addresses provided below. Copies of the software and the User's Manual may also be obtained from these individuals.

Ben Rinehart, Project Manager

Idaho National Engineering and Environmental Laboratorya

P.O. Box 1625, MS 3830

Idaho Falls, ID 83415-3830

(208) 526-1002

Jim Francfort

Idaho National Engineering and Environmental Laboratory

P.O. Box 1625, MS 3830

Idaho Falls, ID 83415-3830

(208) 526-6787

Information concerning the State of New Mexico's involvement with the resource assessment or about the identified sites may be obtained by contacting:

Michael McDiarmid, P.E.

State of New Mexico

Energy, Minerals \& Natural Resources

Department

P.O. Box 6429

Santa Fe, NM 87505-5472

(505) 827-782

a. This work was performed under contract at the Idaho National Engineering Laboratory, with assistance from the State of New Mexico. In January 1997, the name of the INEL was changed to the Idaho National Engineering and Environmental Laboratory (INEEL). INEL will be used on the title page until a new INEEL logo and cover is designed. INEEL will be used throughout the text of the document, except where use of INEL is historically important (for example, p. 1). 


\section{REFERENCES}

Conner, A. M., J. E. Francfort, and B. N. Rinehart, 1996, Uniform Criteria for U.S. Hydropower Resource Assessment, Hydropower Evaluation Software Status Report-II, DOE/ID 10430.1, Idaho National Engineering Laboratory, Idaho Falls, Idaho.

Francfort, J. E., S. D. Matthews, and B. N. Rinehart, 1991, Hydropower Evaluation Software User's Manual, DOE/ID-10338, Idaho National Engineering Laboratory, Idaho Falls, Idaho. 
Appendix A

Summary Report 


\begin{tabular}{|c|c|c|c|c|}
\hline State & $\begin{array}{l}Y \mathrm{D} O \mathrm{P} O \\
\text { Category }\end{array}$ & $\begin{array}{l}\mathrm{R} \quad \mathrm{C} A \\
\text { Number of } \\
\text { Projects }\end{array}$ & $\begin{array}{l}\text { T Y S U M } \\
\text { Name Plate } \\
\text { Capacity (KW) }\end{array}$ & $\begin{array}{l}02 / 04 / 97 \\
\text { R Y } \\
\text { HES Adjusted } \\
\text { Capacity (KW) }\end{array}$ \\
\hline \multirow[t]{4}{*}{$\mathrm{NM}$} & With Power & 2 & 10985 & 5492.50 \\
\hline & w/O Power & 12 & 47889 & 24267.30 \\
\hline & Undeveloped & 8 & 31252 & 5014.80 \\
\hline & STATE TOTAL & 22 & 90126 & 34774.60 \\
\hline \multirow[t]{3}{*}{ TOTALS } & With Power & 2 & 10985 & 5492.50 \\
\hline & w/O Power & 12 & 47889 & 24267.30 \\
\hline & Undeveloped & 8 & 31252 & 5014.80 \\
\hline \multicolumn{2}{|c|}{ ID TOTAI } & 22 & 90126 & 34774.60 \\
\hline
\end{tabular}


Appendix B

River Basins Report 


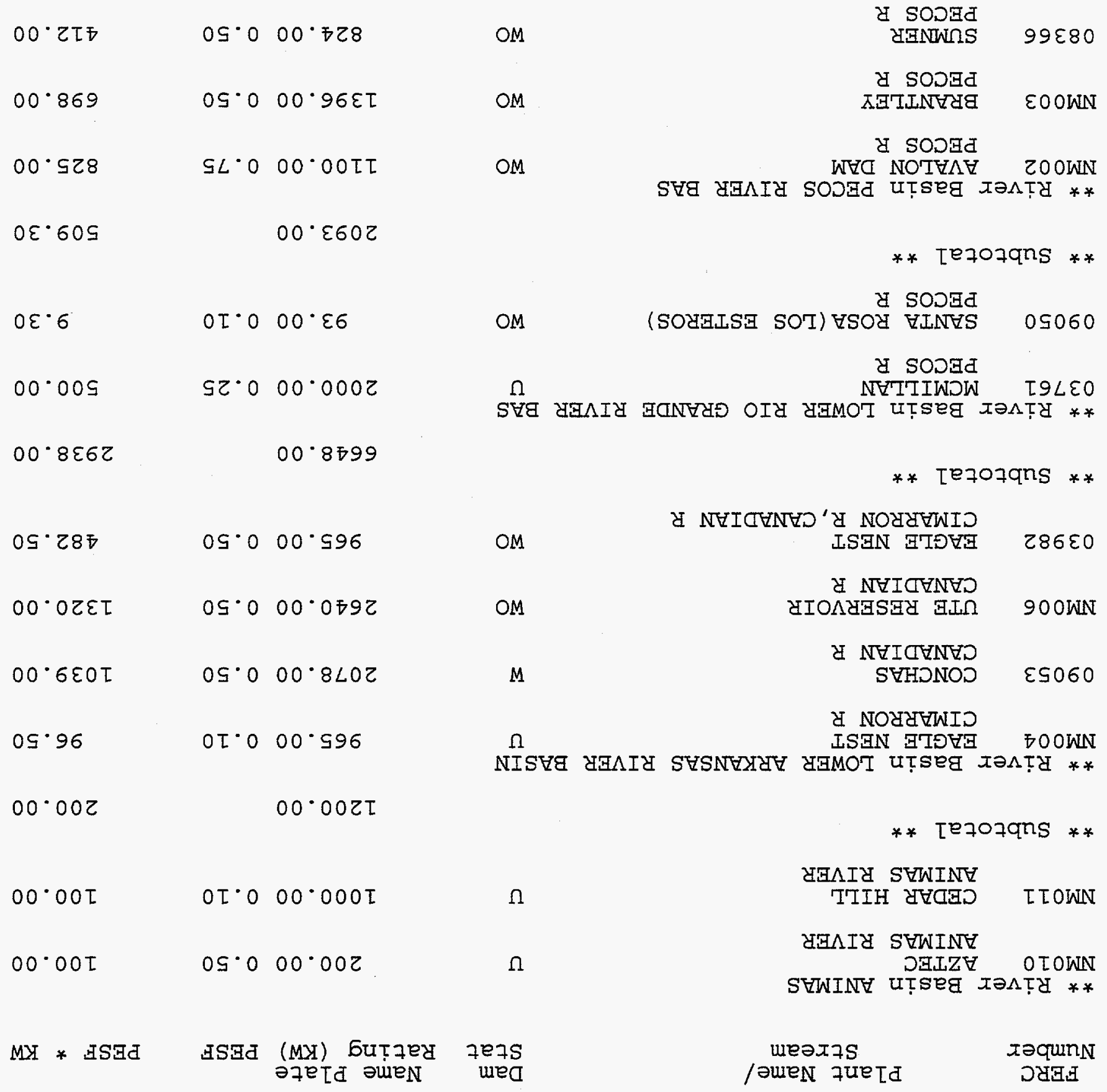

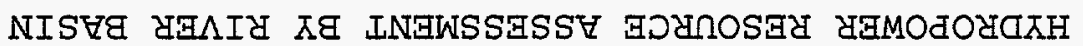

$L 6 / 70 / 20$
$.0 N$ obed


HYDROPOWER RESOURCE ASSESSMENT BY RIVER BASIN

$\begin{array}{cc}\text { FERC } & \text { Plant Name/ } \\ \text { Number } & \text { Stream }\end{array}$
** Subtotal **

* * River Basin RIO GRANDE

NMO0 5 ELEPHANT BUTTE RIO GRANDE

NMO12 BERNALILLO

BERNALILLO CANAL

NMO13 BELEN

NEW BELEN WASTEWAY

** Subtotal **

* * River Basin RIO PENASCO

NMO14 HOPE DIVERSION

RIO PENASCO

** Subtotal **
Dam Name Plate
Stat Rating (KW) PESF PESF * KW

3320.00

1935.00

W

$8907.00 \quad 0.50$

4453.50

wo

$150.00 \quad 0.90$

135.00

wo

$100.00 \quad 0.75$

9157.00

4663.50

U

$1887.00 \quad 0.90$

1698.30

1887.00

1698.30

$11500.00 \quad 0.10$

1150.00

WILLOW CREEK 4

WILLOW CR, RIO CHAMA.

U

U

$8000.00 \quad 0.10$

800.00

$U$

$5700.00 \quad 0.10$

570.00

WILLOW CR, RIO CHAMA

wo

$13630.00 \quad 0.50$

6815.00

RIO GRANDE

wo

$22905.00 \quad 0.50$

11452.50

wo

$86.00 \quad 0.50$

43.00

Wo

$4000.00 \quad 0.50$

2000.00

WILLOW CR, RIO CHAMA

65821.00

22830.50

90126.00 
Appendix C

New Mexico Sites List

C-1 
Page No.

$02 / 04 / 97$

HYDROPOWER RESOURCE ASSESSMENT BY FERC NUMBER

\section{Plant Name/ Stream}

** FERC Number 03761 MCMILLAN PECOS R

* * Subtotal **

* * FERC Number NMOO4 EAGLE NEST

CIMARRON $\mathrm{R}$

* * Subtotal **

** FERC Number NM007 WILLOW CREEK 1

WILLOW CR, RIO CHAMA

* * Subtotal **

* * FERC Number NMO08 WILLOW CREEK 3 WILLOW CR, RIO CHAMA

** Subtotal **

* * FERC Number NM009 WILIOW CREEK 4 WILIOW CR, RIO CHAMA

** Subtotal **

** FERC Number NMO10 AZTEC

ANIMAS RIVER

** Subtotal * *

* * FERC Number NM011 CEDAR HIIL ANIMAS RIVER

$\begin{array}{ll}\text { Dam } & \text { Name Plate } \\ \text { ST Stat } & \text { Rating (KW) PESF }\end{array}$

NM U

$2000.00 \quad 0.25$

2000.00

NM U

$965.00 \quad 0.10$

965.00

96.50

NM U

$5700.00 \quad 0.10$

5700.00

570.00

NM U

$8000.00 \quad 0.10$

8000.00

800.00

NM U

11500.00

0.10

11500.00

1150.00

NM U

$200.00 \quad 0.50$

100.00

200.00

100.00

NM U

$1000.00 \quad 0.10$

100.00 
Page No.

2

$02 / 04 / 97$

HYDROPOWER RESOURCE ASSESSMENT BY FERC NUMBER

$$
\begin{aligned}
& \text { Plant Name/ } \\
& \text { Stream }
\end{aligned}
$$

** Subtotal **

* * FERC Number NMO14

HOPE DIVERSION

RIO PENASCO

** Subtotal **

** FERC Number 09053

CONCHAS

CANADIAN R

* * Subtotal **

* * FERC Number NM005

ELEPHANT BUTTE

RIO GRANDE

** Subtotal **

* * FERC Number 03982

EAGLE NEST

CIMARRON R, CANADIAN R

* * Subtotal **

* * FERC Number 08366

SUMNER

$P E C O S R$

** Subtotal **

* * FERC Number 09050

SANTA ROSA (LOS ESTEROS)

$P E C O S R$

* * Subtotal **

\author{
Dam Name Plate \\ $S T$ Stat Rating (KW) PESF PESF * $\mathrm{KW}$
}

1000.00

100.00

NM U

$1887.00 \quad 0.90$

1698.30

1887.00

1698.30

NM W

$2078.00 \quad 0.50$

1039.00

2078.00

1039.00

NM W

$8907.00 \quad 0.50$

4453.50

8907.00

4453.50

NM WO

$965.00 \quad 0.50$

482.50

965.00

482.50

NM Wo

$824.00 \quad 0.50$

412.00

824.00

412.00

NM wo

$93.00 \quad 0.10$

9.30

93.00

9.30 
Page No.

$02704 / 97$

3

HYDROPOWER RESOURCE ASSESSMENT BY FERC NUMBER

$$
\begin{gathered}
\text { Plant Name/ } \\
\text { Stream }
\end{gathered}
$$

** FERC Number 10890

COCHITI

RIO GRANDE

** Subtotal **

* * FERC Number 10979

HERON

WILLOW CR, RIO CHAMA

** Subtotal **

* * FERC Number 10980

CABALIO

RIO GRANDE

** Subtotal **

** FERC Number NMOOI

AMAIIA (COSTILLA)

COSTILLA CR, RIO GRANDE

* * Subtotal **

* * FERC Number NMOO2

AVALON DAM

PECOS R

* * Subtotal **

** FERC Number NM003

BRANTLEY

PECOS R

** Subtotal **

** FERC Number NM006

UTE RESERVOIR

CANADIAN $R$ $\begin{array}{ll}\text { Dam Name Plate } \\ \text { ST Stat } & \text { Rating (KW) PESF }\end{array}$

NM WO

$22905.00 \quad 0.50$

22905.00

NM wo

NM wo

$13630.00 \quad 0.50$

13630.00

NM WO

NM พO

NM พO

NM Wo
$4000.00 \quad 0.50$

4000.00

$86.00 \quad 0.50$

86.00

43.00

$1100.00 \quad 0.75$

1100.00

$1396.00 \quad 0.50$

1396.00

$\mathrm{PESF}$ * $\mathrm{KW}$

11452.50

11452.50

2000.00

2000.00

6815.00

6815.00

43.00

825.00

825.00

698.00

698.00

$2640.00 \quad 0.50$

1320.00 
Page No.

02/04/97

4

HYDROPOWER RESOURCE ASSESSMENT BY FERC NUMBER

Plant Name/

Stream

** Subtotal **

** FERC Number NM012

BERNAIILIO

BERNALILIO CANAL

** Subtotal **

** FERC Number NM013

BELEN

NEW BELEN WASTEWAY

* * Subtotal **

*** Total ***
Dam Name Plate
Stat Rating (KW) PESF

2640.00

NM WO

$150.00 \quad 0.90$

150.00

NM wo

$100.00 \quad 0.75$

100.00

90126.00
PESF * KW

1320.00

135.00

135.00

75.00

75.00

34774.60 


\section{Appendix D \\ Individual Resource Database List}

D-1 
DATE : $02 / 04 / 97$

PAGE NO: 1

FERC

Number

03761

MCMILIAN

County Name

EDDY

Plant Name

PECOS R

River Basin

LOWER RIO GRANDE RIVER BAS
State

Name

NM

Class Owner Name

$\mathrm{R}$ SEQUOIA ENERGY CORP

Name Plate

PESF

Annual Energy

Rating (KW)

Rating (MWh)

PESF Annual

2000.00

$0.25 \quad 500.00$

5000.00

Energy Rating (MWh)

$\begin{array}{ll}\text { Unit } & \text { Plant } \\ \text { Type } & \text { Type }\end{array}$

C

STG

Factor

wild/Scenic Protection

wild/Scenic Tributary or

Upstream/Downstream

Wild/Scenic Location

Cultural Value

Fish Presence Value

Geologic Value

Historic Value

Other Value

Recreation Value

Scenic Value
Project

Status

$\mathrm{XX}$

Exist Prob

Factor

Exist Prob

0.9 .0

Wildlife Value

Threatened/Endangered Fish

Threatened/Endangered Wildlife

0.90 Federal Land Code 103

0.90 Federal Iand Code 104

0.90 Federal Land Code 105

0.90 Federal Land Code 106

0.90 Federal Land Code 107

0.90 Federal Land Code 108

$Y \quad 0.25$

Federal Land Code 198
1250

3236.00

10421.00 
R E S O U R C E

DATE : 02/04/97

FERC

Number

Plant Name

03982

EAGLE NEST

County Name

COLFAX

Class

Owner Name

M

C.S.

CATTLE

Name Plate

Rating (KW)

PESF

$\mathrm{PESF}^{*} \mathrm{KW}$

Annual Energy Rating (MWh)

2704.00

PESF Annual Energy Rating (MWh)
State

Name

$\mathrm{NM}$
Dam Latitude 3632.00

Status Longitude 10513.00

$\begin{array}{cccll}\text { Unit } & \text { Plant } & \text { Project } & \text { Dam } & \text { Latitude } 3632.00 \\ \text { Type } & \text { Type } & \text { Status } & \text { Status } & \text { Longitude 10513.00 } \\ \text { C } & \text { STG } & \text { XX } & \text { wO } & \end{array}$

Factor

Wild/Scenic Protection

Wild/Scenic Tributary or Upstream/Downstream wild/Scenic Location Cultural Value Fish Presence Value Geologic Value Historic Value other Value Recreation Value Scenic Value
Exist Prob

0.90 Wildlife Value Threatened/Endangered Fish Threatened/Endangered Wildlife

0.90 Federal Land Code 103

0.90 Federal Land Code 104

0.75 Federal Land Code 105

0.90 Federal Land Code 106

0.75 Federal Land Code 107

0.90 Federal Land Code 108

$Y \quad 0.75$ Federal Land Code 198
Exist Prob

0.75

0.90

0.90

0.90

0.75

0.90

0.90

0.90

0.90

0.90 

R E SOURCE
D A T A B A S E
L I S T I N G

DATE : 02/04/97

PAGE NO: 3

FERC

Number

Plant Name

Stream

State

08366 SUMNER

PECOS R

Name

County Name

River Basin

DE BACA

PECOS RIVER BAS

Class Owner Name

R PRODEK, INC

Name Plate

Rating ( $\mathrm{KW}$ )

PESE

Annual Energy

PESF Annual

824.00

$0.50 \quad 412.00$

2400.00

Energy Rating (MWh)

1200

$\begin{array}{cccllr}\text { Unit } & \text { Plant } & \text { Project } & \text { Dam } & \text { Latitude } 3437.00 \\ \text { Type } & \text { Type } & \text { Status } & \text { Status } & \text { Iongitude } 10423.00 \\ \text { C } & \text { ROR } & \text { XX } & \text { Wo } & \end{array}$

Factor

Exist Prob

Factor

Exist Prob

Wild/Scenic Protection

Wild/Scenic Tributary or

Upstream/Downstream

wild/Scenic Location

Cultural Value

Fish Presence Value

Geologic Value

Historic Value

Other Value

Recreation Value

Scenic Value

Exist

0.90 Wildlife Value

Threatened/Endangered Fish

Threatened/Endangered Wildlife 0.90

0.90 Federal Land Code 103

0.90 Federal Land Code 104

$Y \quad 0.75$ Federal Land Code 105

0.90 Federal Land Code 106

0.90 Federal Iand Code 108

$\mathrm{Y} \quad 0.75$

Federal Land Code 198

\begin{tabular}{lll} 
& & \\
\cline { 3 - 3 } Fish & & \\
Wildife & & 0.90 \\
& & 0.75 \\
& & 0.90 \\
& $Y$ & 0.90 \\
& & 0.75 \\
& & 0.90 \\
& & 0.90 \\
& & 0.90 \\
& & 0.90 \\
& & 0.90
\end{tabular}


RESOURC J D D T A B A S E I I S T I N G

DATE : $02 / 04 / 97$

PAGE NO: 4

FERC

Number

Plant Name

Stream

State

Name

09050 SANTA ROSA (LOS ESTEROS) PECOS R

NM

County Name

GUADAIUPE
River Basin

LOWER RIO GRANDE RIVER BAS

Class Owner Name

R SANTA ROSA ASSOC

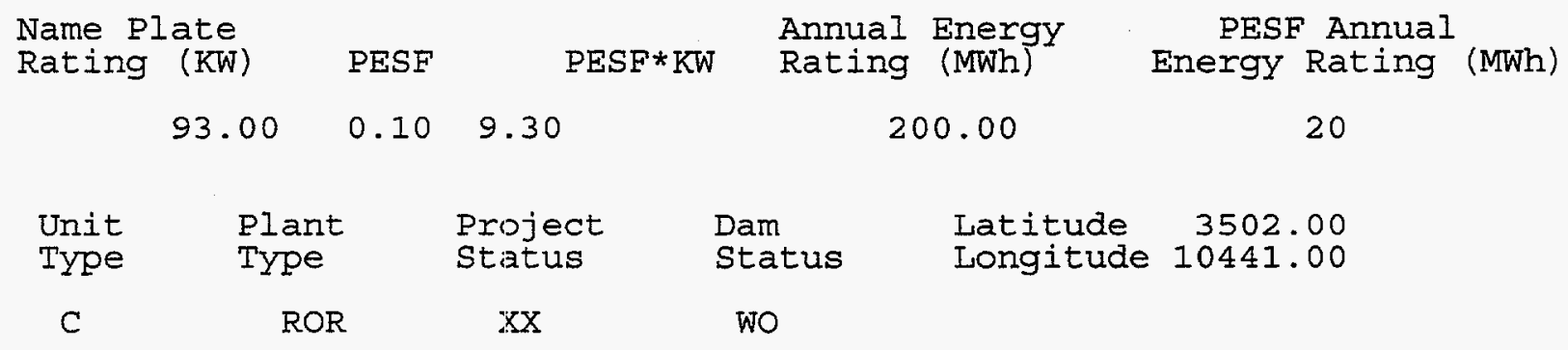

Factor

Wild/Scenic Protection

Wild/Scenic Tributary or Upstream/Downstream wild/Scenic Location Cultural Value

Fish Presence Value

Geologic Value

Historic Value

Other Value

Recreation Value

Scenic Value
Exist Prob

Factor

0.90

$Y$

0.75

0.90

$\mathrm{Y} \quad 0.75$

0.90

$\mathrm{Y} \quad 0.75$

0.90

$\mathrm{Y} \quad 0.75$

$\begin{array}{ll}Y & 0.75 \\ Y & 0.90\end{array}$

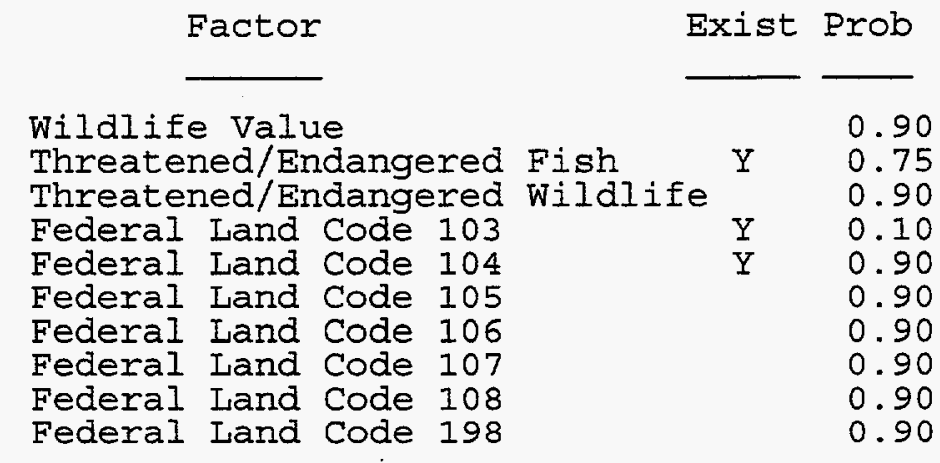


DATE : $02 / 04 / 97$

PAGE NO: 5

FERC

Number

09053
Plant Name CONCHAS

County Name

SAN MIGUEL,
State

Name

NM
CANADIAN R

Stream

River Basin

LOWER ARKANSAS RIVER BASIN
Class Owner Name

$R$ TUCUMCARI ASSOC

Name Plate

Rating (KW)

PESF

Annual Energy

2078.00

0.50

1039.00

$\mathrm{PESF} * \mathrm{KW}$

Rating (MWh)

5900.00

Latitude 3524.00

Longitude 10411.00

$\begin{array}{ccccc}\text { Unit } & \text { Plant } & \text { Project } & \text { Dam } & \text { Latitude } 3524.00 \\ \text { Type } & \text { Type } & \text { Status } & \text { Status } & \text { Longitude 10411.00 } \\ \text { C } & \text { STG } & \text { XX } & \text { W } & \end{array}$

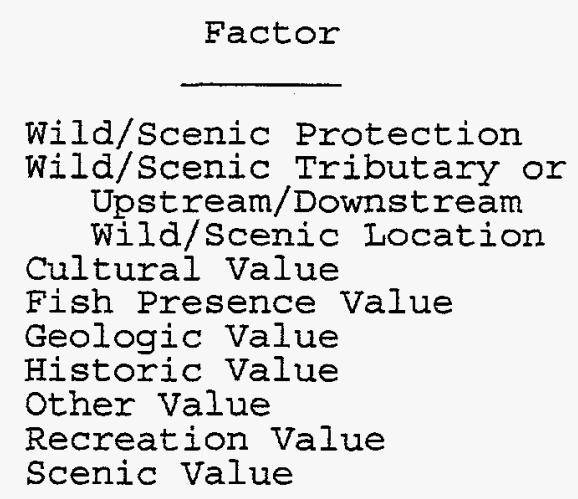

Exist Prob

Factor

Exist Prob

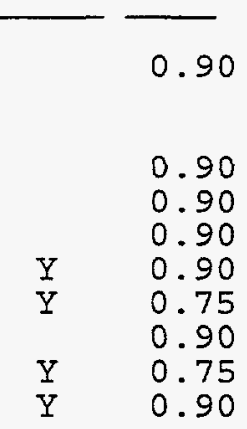

Wildlife value Threatened/Endangered Fish Threatened/Endangered Wildlife Federal Land Code 103 Federal Land Code 104 Federal Land Code 105 Federal Land Code 106 Federal Land Code 107

Federal Land Code 108

Federal Land Code 198 
RESOURCE DA TABASE I I S T I N G

DATE : $02 / 04 / 97$

PAGE NO: 6

FERC

Number

Plant Name

10890

COCHITI

County Name

SANDOVAL,

Stream

State

Name

RIO GRANDE

NM

River Basin

UPPER RIO GRANDE RIVER BAS

Class Owner Name

$\mathrm{R}$ LB INDUSTRIES, INC

\begin{tabular}{|c|c|c|c|c|}
\hline $\begin{array}{l}\text { Name Plate } \\
\text { Rating (KW) }\end{array}$ & PESF & $\mathrm{PESF} * \mathrm{KW}$ & $\begin{array}{l}\text { Annual Energy } \\
\text { Rating (MWh) }\end{array}$ & $\begin{array}{l}\text { PESF Annual } \\
\text { Energy Rating (MWh) }\end{array}$ \\
\hline 22905.00 & 0.50 & 11452.50 & 180000.00 & 90000 \\
\hline
\end{tabular}

$\begin{array}{lllll}\text { Unit } & \text { Plant } & \text { Project } & \text { Dam } & \text { Latitude } 3534.00 \\ \text { Type } & \text { Type } & \text { Status } & \text { Status } & \text { Longitude } 10620.00\end{array}$

C STG XX WO

Factor

Wild/Scenic Protection

Wild/Scenic Tributary or

Upstream/Downstream

wild/Scenic Location

Cultural value

Fish Presence Value

Geologic Value

Historic Value

other Value

Recreation value

Scenic Value
Exist Prob

$\longrightarrow$

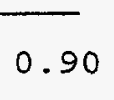

0.90

0.90

$Y \quad 0.75$

0.90

0.90

0.90

$Y$

0.75

0.90
Factor

Wildlife value

Threatened/Endangered Fish

Threatened/Endangered Wildlife

Federal Land Code 103

Federal Land Code 104

Federal Land Code 105

Federal Land Code 106

Federal Land Code 107

Federal Land Code 108

Federal Land Code 198
Exist Prob

0.90

0.75

0.90

0.90

0.75

0.90

0.90

0.90

0.90

0.90 
DATE : $02 / 04 / 97$

PAGE NO: 7

FERC

Number

10979 HERON

County Name

RIO ARRIBA
Plant Name

Class

Owner Name

$\mathrm{R}$ L.B.INDUSTRIES
State Name

\author{
WILLOW CR, RIO CHAMA NM
}

River Basin

UPPER RIO GRANDE RIVER BAS

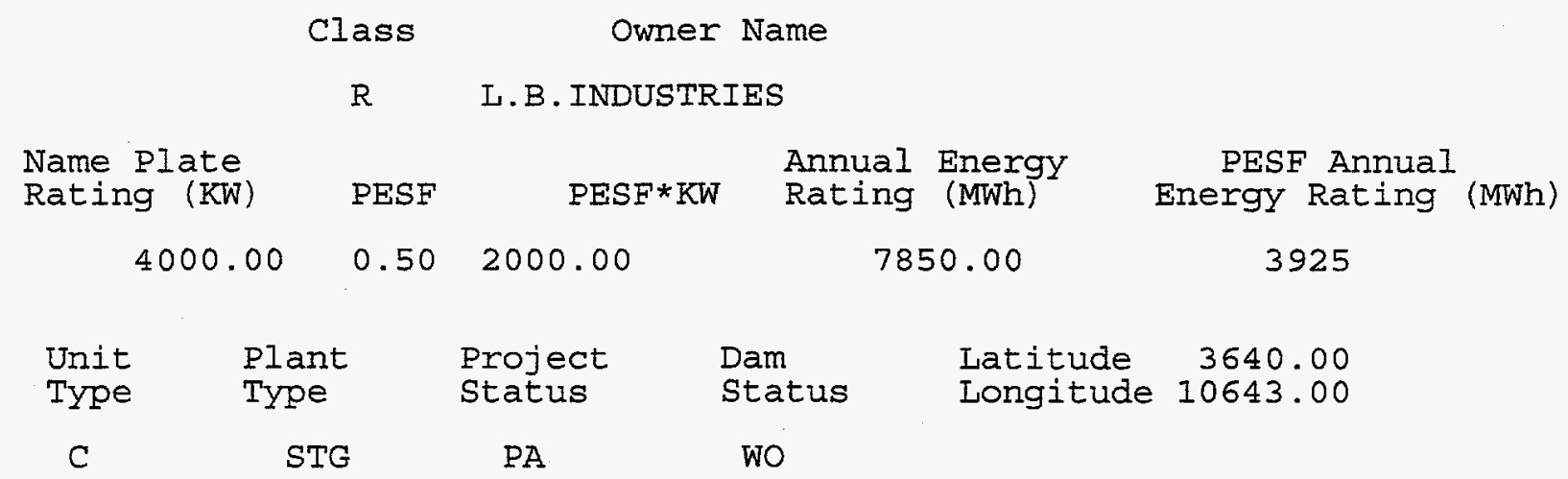

Factor

Wild/Scenic Protection

wild/Scenic Tributary or Upstream/Downstream Wild/Scenic Location Cultural value Fish Presence Value Geologic Value Historic Value Other Value

Recreation Value

Scenic Value

\begin{tabular}{|c|c|c|}
\hline & xist & Prob \\
\hline $\begin{array}{l}\text { Fish } \\
\text { wildlife }\end{array}$ & $\mathrm{Y}$ & $\begin{array}{l}0.90 \\
0.90 \\
0.90 \\
0.90 \\
0.75 \\
0.90 \\
0.90 \\
0.90 \\
0.90 \\
0.90\end{array}$ \\
\hline
\end{tabular}


RES O UR C E: D A T A B A S E I I S T I N G

DATE : $02 / 04 / 97$

PAGE NO: 8

FERC

Number

Plant Name

Stream

State

10980

CABALIO

RIO GRANDE

Name

NM

County Name

River Basin

SIERRA

UPPER RIO GRANDE RIVER BAS

Class

Owner Name

$\mathrm{R}$ L.B. INDUSTRIES, INC

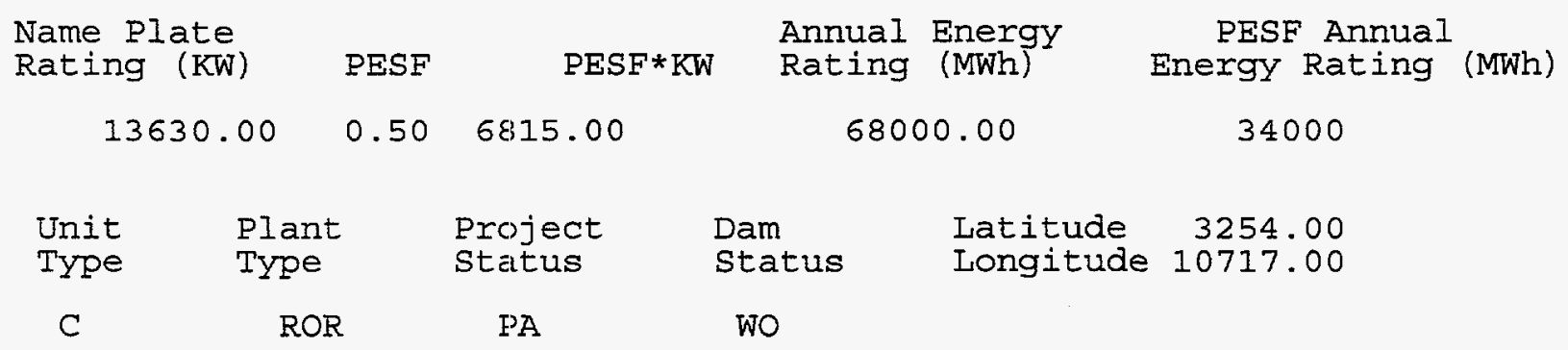

Factor

Wild/Scenic Protection

Wild/Scenic Tributary or Upstream/Downstream wild/scenic Location Cultural Value

Fish Presence Value

Geologic Value

Historic Value

Other Value

Recreation value

Scenic Value
Exist: Prob

-

$\begin{array}{cc} & 0.90 \\ & \\ & \\ & \\ & 0.90 \\ & 0.90 \\ Y & 0.75 \\ & 0.90 \\ & 0.90 \\ & 0.90 \\ & \\ & 0.75 \\ & 0.90\end{array}$

Factor

Exist Prob

Wildlife value Threatened/Endangered Fish Threatened/Endangered WildIife Federal Land Code 103

Federal Land Code 104

Federal Land Code 105

Federal Land Code 106

Federal Land Code 107

Federal Land Code 108

Federal Land Code 198

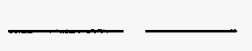

0.90

0.90

0.90

0.90

0.75

0.90

0.90

0.90

0.90

0.90 
DATE : $02 / 04 / 97$

FERC

Number

Plant Name

NM001

AMALIA (COSTILLA)

County Name

TAOS

PAGE NO: 9

State

Name

NM

COSTILIA CR, RIO GRANDE

River Basin

UPPER RIO GRANDE RIVER BAS

Class Owner Name

C RIO COSTILLA COOP. IIVESTOCK ASSOC.

Name Plate
Rating (KW)

86.00

PESF

$0.50 \quad 43.00$

$\mathrm{PESF} * \mathrm{KW}$

Annual Energy

Rating (MWh)

610.00

Energy Rating

PESF Annual

305

$\begin{array}{ll}\text { Unit } & \text { Plant } \\ \text { Type } & \text { Type }\end{array}$

C

STG
Project Status

$\mathrm{XX}$

$\begin{array}{llr}\text { Dam } & \text { Latitude } & 3652.00 \\ \text { Status } & \text { Longitude } & 10517.00\end{array}$

WO
Factor

Wild/Scenic Protection Wild/Scenic Tributary or

Upstream/Downstream

Wild/Scenic Location

Cultural value

Fish Presence Value

Geologic Value

Historic Value

Other Value

Recreation Value

Scenic Value

\begin{tabular}{|c|c|}
\hline xist & Prob \\
\hline $\mathrm{Y}$ & 0.50 \\
\hline$Y$ & $\begin{array}{l}0.90 \\
0.90 \\
0.75 \\
0.90 \\
0.90 \\
0.90\end{array}$ \\
\hline $\begin{array}{l}Y \\
Y\end{array}$ & $\begin{array}{l}0.75 \\
0.90\end{array}$ \\
\hline
\end{tabular}

Factor

Exist Prob

Wildlife value Threatened/Endangered Fish Threatened/Endangered Wildife

Federal Land Code 103

Federal Land Code 104

Federal Land Code 105

Federal Land Code 106

Federal Land Code 107

Federal Land Code 108

Federal Land Code 198
(MWh) 

R E S O U R C E
D A T A B A S E
L I S T I N G

DATE : $02 / 04 / 97$

PAGE NO: 10

FERC

Number

Plant Name

NMOO2
Stream

$P E C O S R$
State

Name

NM
County Name

EDDY
River Basin

PECOS RIVER BAS

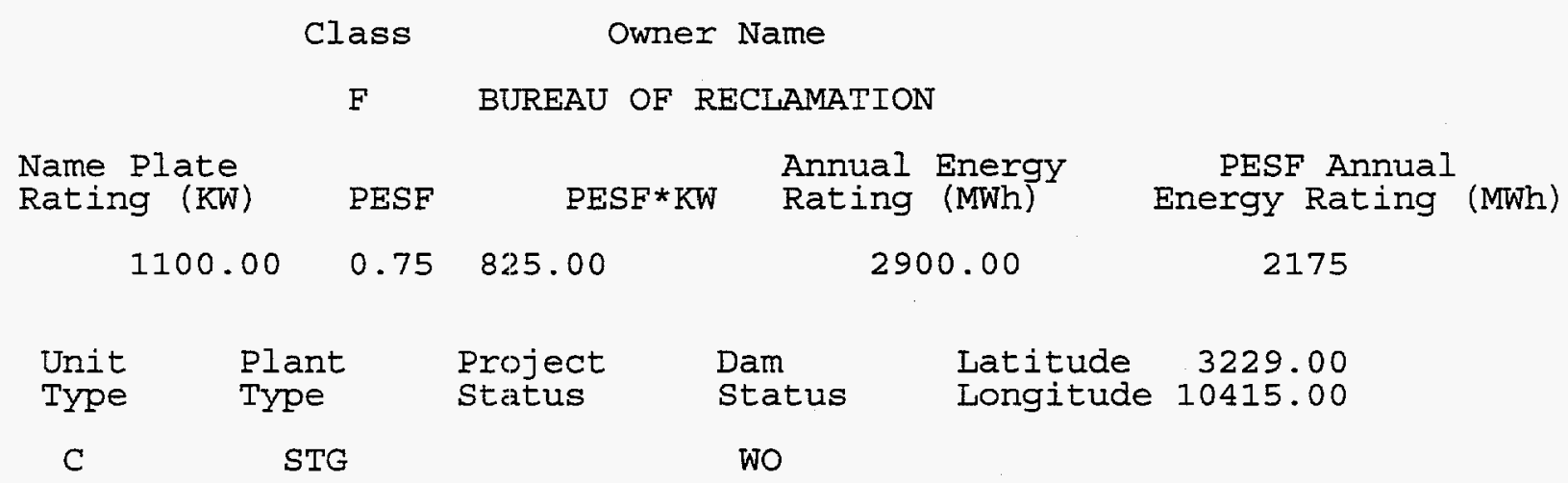

Factor

Wild/Scenic Protection

Wild/Scenic Tributary or Upstream/Downstream wild/Scenic Location Cultural value Fish Presence Value Geologic Value Historic Value Other Value Recreation Value Scenic Value
Exist Prob

0.90

0.90

0.90

0.90

0.90

0.90

0.90

0.90

0.90
Factor

Exist Prob

Wildlife Value Threatened/Endangered Fish Y Federal Land Code 103 Federal Land Code 104 Federal Land Code 105 Federal Land Code 106 Federal Land Code 107 Federal Land Code 108 Federal Land Code 198
0.90

0.75

0.90

0.90

0.90

0.90

0.90

0.90

0.90

0.90 
R E S O U C E D A T A B A E L I S T I N G

DATE : $02 / 04 / 97$

PAGE NO: 11

FERC

Number

Plant Name

Stream

State

Name

NMO03

BRANTIEY

PECOS R

NM

County Name

River Basin

EDDY

PECOS RIVER BAS

Class Owner Name

F BUREAU OF RECILAMATION

Name Plate

PESF

Annual Energy Rating ( $K W)$

$0.50 \quad 698.00$

PESF* KW

Ràting

(MWh)

PESF Annual

1396.00

4390.00

2195

$\begin{array}{cclll}\text { Unit } & \text { Plant } & \text { Project } & \text { Dam } & \text { Latitude } 3232.00 \\ \text { Type } & \text { Type } & \text { Status } & \text { Status } & \text { Longitude } 10416.00 \\ \text { C } & \text { STG } & & \text { wo } & \end{array}$

Factor

Exist Prob

Wild/Scenic Protection

wild/Scenic Tributary or

Upstream/Downstream

wild/Scenic Location

Cultural value

Fish Presence Value

Geologic Value

Historic Value

other Value

Recreation Value

Scenic Value
0.90

Wildlife Value

Threatened/Endangered Fish Threatened/Endangered Wildlife

0.90

Federal Land Code 103

0.90 Federal Land Code 104

0.90 Federal Land Code 105

0.90 Federal Land code 106

0.90 Federal Land Code 107

0.90 Federal Land Code 108

$Y \quad 0.75$

0.90
Exist Prob

0.90

0.75

0.90

0.90

0.90

0.90

0.90

0.90

0.90

0.90 
RES O URCE D A T A B A S E I I S T I N G

DATE : $02 / 04 / 97$

PAGE NO: 12

FERC

Number

Plant Name

Stream

State

NMO04 EAGLE NEST

CIMARRON R

Name

NM

County Name

COLFAX
River Basin

IOWER ARKANSAS RIVER BASIN

Class Owner Name

$R$ C.S. CATTLE COMPANY

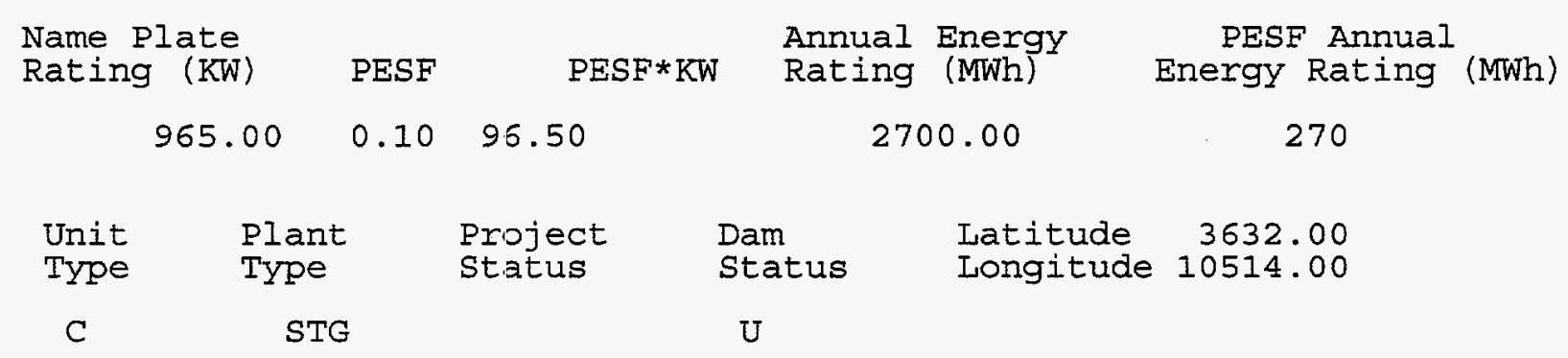

\section{Factor}

Wild/Scenic Protection

Wild/Scenic Tributary or Upstream/Downstream wild/scenic Location Cultural value Fish Presence Value Geologic Value Historic Value Other Value Recreation Value Scenic Value
Exist Prob

\begin{tabular}{|c|c|}
\hline & 0.90 \\
\hline$Y$ & $\begin{array}{l}0.90 \\
0.90 \\
0.25 \\
0.90 \\
0.90 \\
0.90\end{array}$ \\
\hline $\begin{array}{l}Y \\
Y\end{array}$ & $\begin{array}{l}0.25 \\
0.50\end{array}$ \\
\hline
\end{tabular}

Factor

Wildlife Value Threatened/Endangered Fish Threatened/Endangered Wildlife Exist Prob Federal Land Code 103

Federal Land Code 104

Federal Land Code 105

Federal Land Code 106

Federal Land Code 107

Federal Land Code 108

Federal Land Code 198 
$R E S O U R C E$

DATE : $02 / 04 / 97$
D A T A B A S E

I I S T I N G

PAGE NO: 13

FERC

Number

Plant Name

Stream

State

NMO0 5

ELEPHANT BUTTE

County Name

RIO GRANDE

$\mathrm{NM}$

River Basin

SIERRA

RIO GRANDE

Class Owner Name

F BUREAU OF RECLAMATION

Name Plate

Rating ( $\mathrm{KW}$ )

PESF

Annual Energy

8907.00

$0.50 \quad 4453.50$

PESF Annual

Energy Rating (MWh)

Unit

Type

C
Plant
Type

STG

Factor

Wild/Scenic Protection

Wild/Scenic Tributary or Upstream/Downstream Wild/Scenic Location

Cultural Value

Fish Presence Value

Geologic Value

Historic Value

Other Value

Recreation Value

Scenic Value
Exist Prob

0.90

Wildife Value

Threatened/Endangered Fish

Threatened/Endangered Wildlife

0.90

0.90

Federal Land Code 103

Federal Land Code 104

Federal Land Code 105

Federal Land Code 106

0.90

0.90

0.90

$Y \quad 0.75$

0.90
Federal Land Code 107

Federal Land Code 108

Federal Land Code 198
4143.00

2071.50

$\begin{array}{lr}\text { Latitude } & 3309.00 \\ \text { Longitude } & 10711.00\end{array}$

W
0.90

0.90

0.90

0.90

0.90

0.90

0.90

0.90

0.90

0.90 
RE S O URC E D A T A B A S E I I S T I N G

DATE : $02 / 04 / 97$

PAGE NO: 14

FERC

Number

Plant Name

Stream

State

NM006 UTE RESERVOIR

CANADIAN $R$

Name

\section{NMOO6 UTE RESERVOIR}

County Name

QUAY

River Basin

LOWER ARKANSAS RIVER BASIN

\section{Class Owner Name}

F BUREAU OF RECIAAMATION

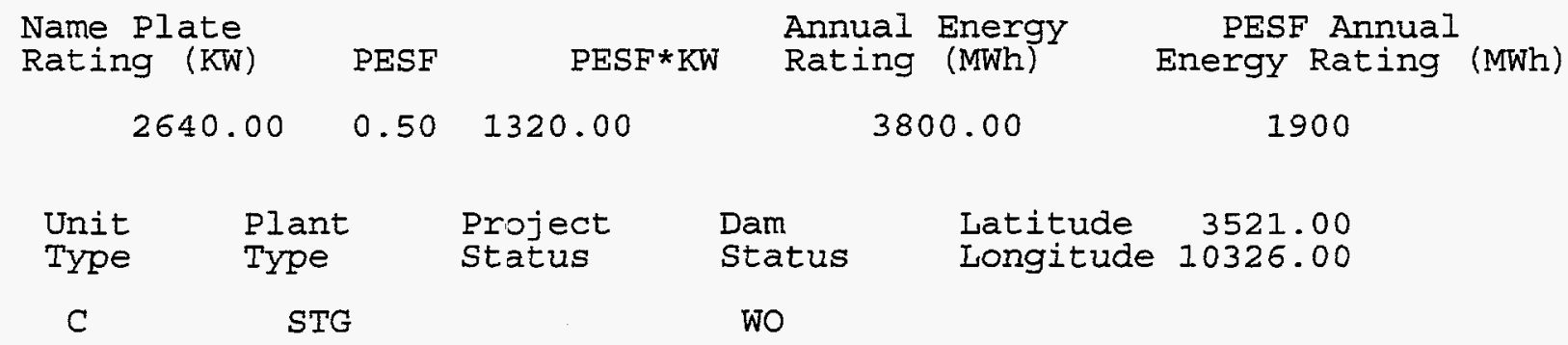

Factor

Wild/Scenic Protection

Wild/Scenic Tributary or Upstream/Downstream wild/Scenic Location Cultural Value

Fish Presence Value

Geologic Value

Historic Value

Other Value

Recreation value

Scenic value
Exist Prob

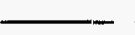

0.90

Wildlife Value

Threatened/Endangered $\mathrm{Fish}$ Threatened/Endangered Wildlife

0.90 Federal Land Code 103

0.90 Federal Land Code 104

0.90 Federal Land Code 105

0.90 Federal Land Code 106

0.90 Federal Land Code 107

0.90
0.75 Federal Land Code 108

Y $\quad 0.75$
NM 
DATE: $02 / 04 / 97$

PAGE NO: 15

FERC

Number

Plant Name

NMO07

WILLOW CREEK 1

County Name

RIO ARRIBA

Class

Owner Name
State

Name

NM
River Basin

UPPER RIO GRANDE RIVER BAS

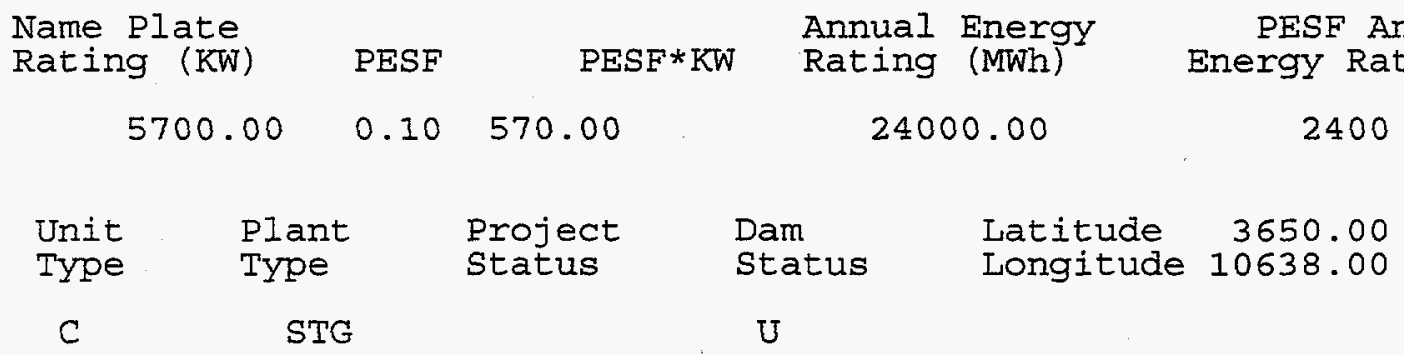

Factor

Wild/Scenic Protection wild/Scenic Tributary or Upstream/Downstream wild/scenic Location Cultural value Fish Presence Value Geologic Value Historic Value Other Value Recreation Value Scenic Value
Exist Prob

$\mathrm{Y}$

0.10

Wildlife Value Threatened/Endangered Fish Threatened/Endangered Wildlife

Exist Prob

$\begin{array}{ll}0.90 & \text { Federal Land Code } 103 \\ 0.90 & \text { Federal Land Code } 104\end{array}$

0.90 Federal Land Code 105

0.90 Federal Land Code 106

0.90 Federal Land Code 107

0.90 Federal Land Code 108

0.90 Federal Land Code 198

0.90
0.90

0.90

0.90

0.90

0.90

0.90

0.90

0.90

0.90

0.90 
RESOURCE D A T A B A S E I I S T I N G

DATE : $02 / 04 / 97$

PAGE NO: 16

FERC

Number

Plant Name

NM008 WILLOW CREEK 3

County Name

RIO ARRIBA

\section{Class}

Name Plate Rating (KW) PESF

8000.00

$$
0.10800 .00
$$

$\mathrm{PESF} * \mathrm{KW}$

Annual Energy Rating (MWh)

$$
58000.00
$$

Unit Plant

Type

Type

Project status

C

STG

\section{Factor}

Wild/Scenic Protection Wild/Scenic Tributary or Upstream/Downstream wild/Scenic Location Cultural value

Fish Presence Value

Geologic Value

Historic Value

other Value

Recreation Value

Scenic Value
Exist Prob

$Y$

0.10

Wildlife Value

Threatened/Endangered Fish Threatened/Endangered Wildlife

0.90 Federal Iuand Code 103

0.90 Federal Irand Code 104

0.90 Federal Iand Code 105

0.90 Federal Iuand Code 106

0.90 Federal Luand Code 107

0.90 Federal Land Code 108

0.90 Federal Land Code 198

0.90 $\begin{array}{llr}\text { Dam } & \text { Latitude } & 3644.00 \\ \text { Status } & \text { Longitude } & 10637.00\end{array}$

U
State Name

NM
PESF Annual
Energy Rating (MWh)

\section{0}


$R E S O U R C E$

DATE : $02 / 04 / 97$

FERC

Number

Plant Name

NMOO 9

WILLOW CREEK 4

County Name

RIO ARRIBA

Class

Owner Name
L I S T I N G

PAGE NO: 17

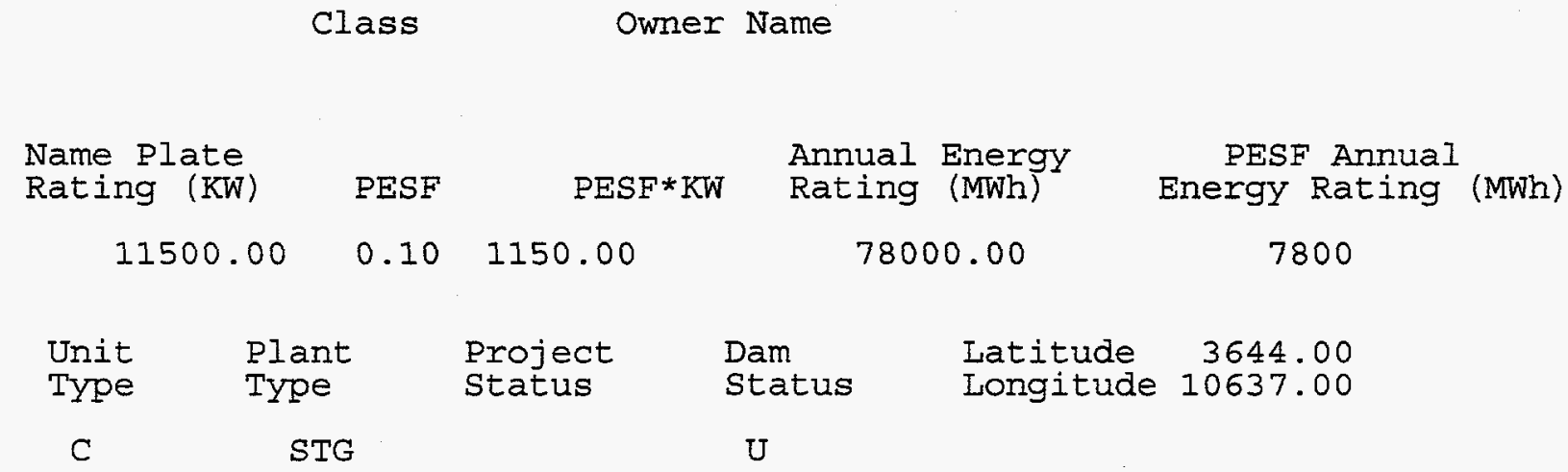

Factor

Wild/scenic Protection

wild/Scenic Tributary or Upstream/Downstream Wild/Scenic Location Cultural value

Fish Presence Value

Geologic Value

Historic Value

other Value

Recreation Value

scenic Value
Exist Prob

0.10

$\mathrm{Y}$

0.10

Wildlife Value

Threatened/Endangered Fish

Threatened/Endangered Wildlife

Exist Prob

0.90

0.90

Federal Land Code 103

0.90

0.90

0.90

0.90

0.90

0.90
Federal Land Code 104

Federal Land Code 105

Federal Land Code 106

Federal Land Code 107

Federal Land Code 108

Federal Land Code 198

\begin{tabular}{ll} 
Exist & Prob \\
\cline { 3 - 3 } Fish & \\
\cline { 3 - 3 } Wildife & 0.90 \\
& 0.90 \\
& 0.90 \\
& 0.90 \\
& 0.90 \\
& 0.90 \\
& 0.90 \\
& 0.90 \\
& 0.90 \\
& 0.90
\end{tabular}

State

Name

NM 
RESOURC I D D TABASE I I S T I N G

DATE : $02 / 04 / 97$

PAGE NO: 18

FERC

Number

Plant Name

Stream

State

NM010 AZTEC

ANIMAS RIVER

Name

$\mathrm{NM}$

County Name

River Basin

SAN JUAN

ANIMAS

$\begin{array}{cr}\text { Class } & \text { Owner Name } \\ \text { M } & \text { CITY OF AZTEC }\end{array}$

Name Plate

Rating (KW)

$\begin{array}{ll} & \text { Annual Energy } \\ \text { PESF } & \text { PESF*KW } \\ \text { Rating }(\mathrm{MWh})\end{array}$

PESF Annual

200.00

0.50100 .00

1685.00

842.50

$\begin{array}{lllll}\text { Unit } & \text { Plant } & \text { Project } & \text { Dam } & \text { Latitude } 3649.00 \\ \text { Type } & \text { Type } & \text { Status } & \text { Status } & \text { Longitude 10801.00 }\end{array}$

C

ROR

$\mathrm{ZZ}$

Exist Prob

Factor

Wild/Scenic Protection

Wild/Scenic Tributary or Upstream/Downstream Wild/Scenic Location

Cultural value

Fish Presence Value

Geologic Value

Historic Value

Other value

Recreation Value

Scenic Value
Status

$\mathrm{U}$
Energy Rating (MWh) 

R E SOU R
C E
D A T A B A S E
I I S T I N G

DATE : $02 / 04 / 97$

PAGE NO: 19

FERC

Number

NM011 CEDAR HIIL

County Name

SAN JUAN

$$
\text { Name }
$$

Plant Name

Class

M

\section{CITY}

Owner Name

Name Plate

Rating (KW)

PESF

0.10

100.00
PESF* Annual Energy
Rating (MWh)

8425.00

ANIMAS RIVER

Stream

State

Name

NM
River Basin

ANIMAS
Plant

Type

C

Factor

Wild/Scenic Protection

Wild/Scenic Tributary or Upstream/Downstream wild/Scenic Location Cultural value

Fish Presence Value

Geologic Value

Historic Value

other value

Recreation Value

Scenic Value

\begin{abstract}
Project status
\end{abstract}

$\mathrm{XX}$

Exist Prob

0.90

Wildife Value

Threatened/Endangered Fish

Threatened/Endangered Wildlife

$\begin{array}{llr}\text { Dam } & \text { Latitude } & 3656.00 \\ \text { Status } & \text { Longitude } & 10754.00\end{array}$ $\begin{array}{llr}\text { Dam } & \text { Latitude } & 3656.00 \\ \text { Status } & \text { Longitude } & 10754.00\end{array}$

$\mathrm{U}$
PESF Annual

Energy Rating (MWh)

842.50 
DATE : $02 / 04 / 97$

PAGE NO: 20

FERC

Number

Plant Name

NMO 12

BERNAIILLO

County Name

BERNALILLO
Stream

BERNALILLO CANAL

River Basin

RIO GRANDE
State

Name

NM

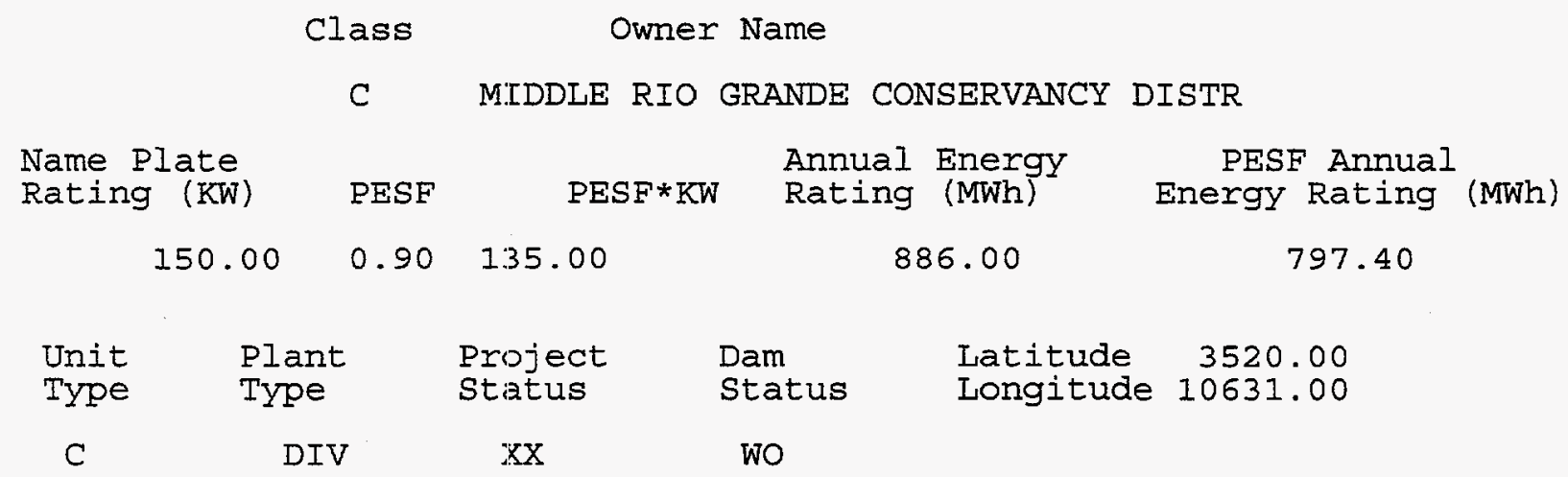

Factor

wild/Scenic Protection

Wild/Scenic Tributary or Upstream/Downstream wild/Scenic Location Cultural Value

Fish Presence Value

Geologic Value

Historic Value

Other Value

Recreation Value

Scenic Value
Exist Prob

0.90 Threatened/Endangered Fish Threatened/Endangered Wildlife

0.90

0.90

0.90

0.90

0.90

0.90

0.90

0.90
Wildlife Value

Factor

Tera 1 Iand code 103

Federal Land Code 104

Federal Land Code 105

Federal Land Code 106

Federal Land Code 107

Federal Land Code 108

Federal Land Code 198
Exist Prob

0.90

0.90

0.90

0.90

0.90

0.90

0.90

0.90

0.90

0.90 
DATE : $02 / 04 / 97$

FERC

Number

Plant Name

NM013 BELEN

County Name

VALENCIA
PAGE NO: 21

State

Name

NM
NEW BELEN WASTEWAY

River Basin

RIO GRANDE

Class Owner Name

C MIDDLE RIO GRANDE CONSERVANCY DIST.

\begin{tabular}{|c|c|c|c|c|c|c|c|}
\hline $\begin{array}{l}\text { Name } \mathrm{P} \\
\text { Rating }\end{array}$ & $\begin{array}{l}\text { late } \\
(\mathrm{KW})\end{array}$ & PESF & $\mathrm{PE}$ & & $\begin{array}{l}\text { Annual } \\
\text { Rating }\end{array}$ & $\begin{array}{l}\text { Energy } \\
(\mathrm{MWh})\end{array}$ & $\begin{array}{c}\text { PESF Ar } \\
\text { Energy Rat }\end{array}$ \\
\hline & 100.00 & 0.75 & 75.00 & & & 25.00 & 393 \\
\hline $\begin{array}{l}\text { Unit } \\
\text { Type }\end{array}$ & $\begin{array}{l}\text { Pla } \\
\text { Tyl }\end{array}$ & & $\begin{array}{l}\text { Project } \\
\text { Status }\end{array}$ & & atus & $\begin{array}{l}\text { Latitude } \\
\text { Longitude }\end{array}$ & $\begin{array}{r}3438.00 \\
10647.00\end{array}$ \\
\hline $\mathrm{C}$ & & & $\mathrm{XX}$ & & 10 & & \\
\hline
\end{tabular}

Factor

Wild/Scenic Protection

Wild/Scenic Tributary or

Upstream/Downstream

wild/Scenic Location

Cultural value

Fish Presence Value

Geologic Value

Historic Value

other value

Recreation Value

Scenic Value
Exist Prob

0.90

Wildlife value

Threatened/Endangered Fish Threatened/Endangered Wildlife

0.90 Federal Land Code 103

0.90 Federal Land Code 104

0.90 Federal Land Code 105

0.90 Federal Land Code 106

0.90 Federal Land Code 107

0.90 Federal Land Code 108

Y

0.75

0.90
Exist Prob

0.90

0.90

0.90

0.90

0.90

0.90

0.90

0.90

0.90

0.90 

R ESOU R C E
D A T A B A S E
I I S T I N G

DATE : $02 / 04 / 97$

PAGE NO: 22

FERC

Number

Plant Name

Stream

State

NMO14 HOPE DIVERSION

RIO PENASCO

Name

County Name

River Basin

CHAVEZ

RIO PENASCO

Class Owner Name

C HOPE WATER USERS ASSOCIATION

Name Plate

PESF $\quad$ PESF* KW $^{*} \begin{aligned} & \text { Annual Energy } \\ & \text { Rating (MWh) }\end{aligned}$

PESF Annual

Rating (KW)

$0.90 \quad 1698.30$

11812.00

Energy Rating

(MWh)

1887.00

Project

Dam

Status

Latitude $\quad 3250.00$

Type

Type

status U

NM

c

DIV

$\mathrm{XX}$

Factor

Wild/Scenic Protection

wild/Scenic Tributary or Upstream/Downstream wild/Scenic Location

Cultural Value

Fish Presence Value

Geologic Value

Historic value

Other Value

Recreation Value

Scenic Value
Exist Prob

0.90

wildlife Value

Threatened/Endangered Fish

Threatened/Endangered Wildlife

0.90 Federal Land Code 103

0.90 .90 Federal Land Code 104

0.90 Federal Land Code 105

0.90 Federal Land Code 106

0.90 Federal Land Code 107

0.90 Federal Land Code 108

0.90 Federal Land Code 198

0.90
10630.80

0456.00 